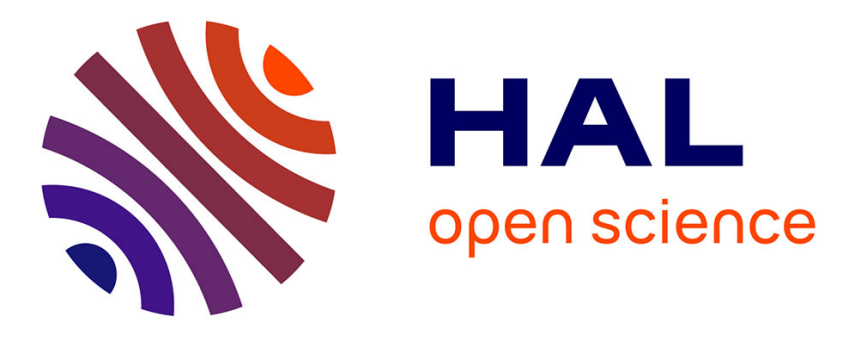

\title{
Ratio-Based Multitemporal SAR Images Denoising: RABASAR
}

Weiying Zhao, Charles-Alban Deledalle, Loïc Denis, Henri Maître, Jean-Marie Nicolas, Florence Tupin

\section{- To cite this version:}

Weiying Zhao, Charles-Alban Deledalle, Loïc Denis, Henri Maître, Jean-Marie Nicolas, et al.. RatioBased Multitemporal SAR Images Denoising: RABASAR. IEEE Transactions on Geoscience and Remote Sensing, 2019, 10.1109/TGRS.2018.2885683 . hal-01791355v2

\section{HAL Id: hal-01791355 \\ https://hal.science/hal-01791355v2}

Submitted on 26 Nov 2018

HAL is a multi-disciplinary open access archive for the deposit and dissemination of scientific research documents, whether they are published or not. The documents may come from teaching and research institutions in France or abroad, or from public or private research centers.
L'archive ouverte pluridisciplinaire HAL, est destinée au dépôt et à la diffusion de documents scientifiques de niveau recherche, publiés ou non, émanant des établissements d'enseignement et de recherche français ou étrangers, des laboratoires publics ou privés. 


\title{
Ratio-based Multi-temporal SAR Images Denoising: RABASAR
}

\author{
Weiying Zhao, Charles-Alban Deledalle, Loïc Denis, Henri Maître, Jean-Marie Nicolas, \\ Florence Tupin, Senior Member, IEEE
}

\begin{abstract}
In this paper, we propose a fast and efficient multitemporal despeckling method. The key idea of the proposed approach is the use of the ratio image, provided by the ratio between an image and the temporal mean of the stack. This ratio image is easier to denoise than a single image thanks to its improved stationarity. Besides, temporally stable thin structures are well preserved thanks to the multi-temporal mean.

The proposed approach can be divided into three steps: 1) estimation of a "super-image" by temporal averaging and possibly spatial denoising; 2) denoising of the ratio between the noisy image of interest and the "super-image"; 3) computation of the denoised image by re-multiplying the denoised ratio by the "super-image".

Because of the improved spatial stationarity of the ratio images, denoising these ratio images with a speckle-reduction method is more effective than denoising images from the original multi-temporal stack. The amount of data that is jointly processed is also reduced compared to other methods through the use of the "super-image" that sums up the temporal stack. The comparison with several state-of-the-art reference methods shows better results numerically (peak signal-noise-ratio, structure similarity index) as well as visually on simulated and SAR time series. The proposed ratio-based denoising framework successfully extends single-image SAR denoising methods to time series by exploiting the persistence of many geometrical structures.
\end{abstract}

Index Terms-Multi-temporal SAR series, ratio image, superimage, $\mathrm{SAR}$, speckle reduction

\section{INTRODUCTION}

$\mathbf{S}$ YNTHETIC aperture radar (SAR) imaging is widely used in the monitoring of land surfaces, disasters or the environment, due to its all-time acquisition capability, its sensitivity to geometric structures, its penetration characteristics, etc. However, the system-inherent speckle noise visually corrupts the appearance of images and severely diminishes the analysis and interpretation of SAR images. Therefore, a preliminary speckle reduction step is often necessary for the successful exploitation of SAR images.

The recent and unprecedented availability of long time series with Sentinel-1 constellation has opened new ways for SAR speckle reduction. This highly redundant information offers a new paradigm. Taking inspiration from temporal multi-looking and residual noise analysis we propose in this

W. Zhao, H. Maître, J.-M. Nicolas, F. Tupin are with LTCI, Télécom ParisTech, Université Paris-Saclay, 75013 Paris, France

L. Denis is with Univ Lyon, UJM-Saint-Etienne, CNRS, Institut d Optique Graduate School, Laboratoire Hubert Curien UMR 5516, F-42023, SAINTETIENNE, France

C.-A. Deledalle is with IMB, CNRS, Univ. Bordeaux, Bordeaux INP, F33405 Talence, France paper a ratio-based denoising approach (first presented in [1]), dedicated to long multi-temporal stacks.

The main problems of SAR image despeckling are the spatial resolution preservation, the edges and textures restoration, and the preservation of point-like targets. Spatial multilooking is a common way to reduce speckle fluctuations in a single SAR image, at the cost of a significant spatial resolution loss [2]. To effectively estimate the noise-free reflectivity as well as preserve the spatial resolution, many single-channel SAR speckle reduction methods have been proposed during the past decades. Detailed introductions of the methods are given by Touzi et al. [3], Argenti et al. [2] and Deledalle et al. [4]. These methods mainly belong to four categories: Bayesian methods in the spatial domain (Lee filter [5], Lee refined filter [6]), Bayesian methods in a transformed domain [7], selection-based filtering (IDAN [8], PPB [9] and NLSAR [10]) and sparse-based approaches [11]. Many singleSAR-image denoising methods are designed by combining information in different domains (the spatial domain and a transformed domain), with different estimation criteria and various statistical models of speckle and radar reflectivities [2]. Several state-of-the-art single-image despeckling methods perform a weighted average of selected surrounding pixels values to estimate the speckle-free values. If the surrounding pixels are not well selected or if their associated weights are badly chosen, an estimation bias occurs which is generally visible in the form of a spatial resolution loss (over-smoothing and spreading of thin structures). The accurate selection of pixels is very challenging in single-look SAR images given the large fluctuations due to speckle phenomenon. Even when using state-of-the-art spatial denoising approaches (such as SAR-BM3D [12] or NL-SAR [10]), the smallest and least contrasted structures can be damaged or some noticeable speckle fluctuations can remain after filtering.

Recently, convolutional neural networks have shown a high capability of denoising data affected by additive white Gaussian noise (AWGN) [13]. Application to SAR images has also been proposed, either through an homomorphic approach [14] or directly applying gamma distribution based methods [15]. Unlike traditional SAR image denoising approaches, these methods predict the noise-free value through the estimation of the speckle component. These recent techniques reach comparable despeckling results w.r.t. state-of-the-art approaches, both in terms of signal to noise ratio (simulated data) and in terms of spatial feature preservation. However, the training of these networks is time consuming and requires to building a training set with pairs of speckle-free / speckled SAR images. 
With the launch of the latest generation of SAR satellites (Cosmo-SkyMed, TerraSAR-X, ALOS-2, Sentinel-1, etc.) [16], more and more SAR images, with shorter revisit time or higher resolution, are now available. With multi-temporal images, both spatial and temporal information can be combined in the denoising process, which gives the potential of reaching better speckle reduction results than is achievable when processing a single image. This is the path followed by several kinds of multi-temporal denoising methods, proposing temporal weighted average [17], [18], [19], temporal weighted average in a transformed domain (M-TSF [20] and MSARBM3D [21]), change-detection-aware multi-temporal average (Lê's method [22], [23] and 2SPPB [24]), or filtering using three-dimensional adaptive neighborhoods [25], [26]. A drawback of such approaches is the increased computational complexity with longer time series.

Multi-temporal denoising methods take advantage of the increasing availability of SAR image time-series to solve the spatial denoising problems, for the benefit of a better spatial resolution preservation. Most of multi-temporal denoising methods process the whole time series in order to produce a denoised image at a given date. In this paper, we take a different approach by forming a summary of the multitemporal series (which we call a "super-image", the speckle in that image being strongly reduced), and by using only this "super-image" in addition to the speckle-corrupted image (rather than the whole time series) to obtain a denoised image at any given date.

This ratio-based multi-temporal denoising method fully exploits the significant information of the multi-temporal stack through the "super-image". After forming the ratio image between the noisy image and the "super-image", the proposed method takes advantage of the state-of-the-art single-image speckle reduction methods to denoise the ratio image [1]. We consider multi-temporal images acquired on the same orbit (i.e., either all ascending orbits, or all descending orbits), with similar incidence angles, and which have been accurately registered [27]. For the sake of reproducible research, we have released an open-source code at https://www.math.u-bordeaux. fr/ cdeledal/rabasar.php.

The contributions of this paper are the following:

- we introduce a generic ratio-based multi-temporal denoising framework, called RABASAR, to process a single SAR image using a multi-temporal stack;

- we design a denoiser, called RuLoG, adapted to the statistical distribution of the ratio image formed between a speckle-corrupted image and a "super-image" with reduced speckle, by extending the MuLoG method proposed in [28];

- we demonstrate the interest of the proposed method on simulated and SAR time series.

The remainder of this paper is organized as follows. In Sec. II, we introduce the general framework of the proposed method. Section III presents different ways to compute the "super-image". Then the filtering of the ratio image is described in Sec.IV. Experimental results are presented in Sec. V. Finally, some conclusions and perspectives are drawn in Sec. VI.

\section{PRINCIPLE OF THE PROPOSED METHOD}

The temporal averaging (also called temporal multi-looking) of SAR time series produces an image with reduced speckle and a preserved spatial resolution. We call such a reducedspeckle image a "super-image". In this paper, we propose to exploit this super-image to build a ratio-based denoising framework.

The proposed method is composed of three main steps, illustrated in Fig. 1:

1) In the first step, a super-image is calculated from a time series of spatially registered and radiometrically calibrated SAR images. Averaging temporal intensity samples is the simplest way to obtain an image with reduced speckle. This corresponds to the maximum likelihood estimation of the reflectivity when speckle is considered temporally decorrelated and temporal fluctuations of the reflectivity are neglected (observations at a given pixel are then independent and identically distributed). In practice, changes impacting some regions of the image may occur at some dates. Rather than averaging all dates, it can be beneficial to select only relevant dates when forming the "super-image", as done for instance in [24], [23], [29]. Moreover, because speckle is temporally correlated (by spatially varying correlation levels), some amount of spatial denoising helps to produce a "super-image" where speckle is reduced everywhere. Computation of the super-image, denoted $\hat{u}_{m}$ in the sequel, will be presented in Sec. III.

2) In the second step, the super-image is used to form the ratio $\tau_{t}$ between the image $v_{t}$ at time $t$ and the super-image, at each spatial location $s$ :

$$
\tau_{t}(s)=\frac{v_{t}(s)}{\hat{u}_{m}(s)}
$$

We refer to $\tau_{t}$ as the "ratio image" at time $t$. It contains the residual speckle noise between the two images, and the radiometric shifts when changes occur. When the length of the time series increases and in the absence of change, the superimage $\hat{u}_{m}$ converges to the reflectivity $u_{t}$. The ratio image $\tau_{t}$ then tends to pure speckle (i.e., a collection of independent identically distributed gamma random variables with unitary mean and the same number of looks as the original image). In contrast, when changes occur in the time series, these changes impact the super-image which then differs from the reflectivity $u_{t}$ of the image at time $t$. Processing the ratio image $\tau_{t}$ is necessary to correctly recover the reflectivity $u_{t}$. Both the noisy image $v_{t}$ and the super-image $\hat{u}_{m}$ suffer from speckle (the speckle in the super-image, though, is strongly reduced). The processing of the ratio image requires to account for the specific noise statistics arising from this ratio of speckled images. A denoiser dedicated to the statistics of the ratioimage is derived in Sec. IV.

3) In the third step, the filtered image is recovered by multiplying the denoised ratio image with the original superimage.

\section{Computation of the Super-IMAge}

The super-image can be computed from a time series by different ways. First, different Hölder means (such as the 


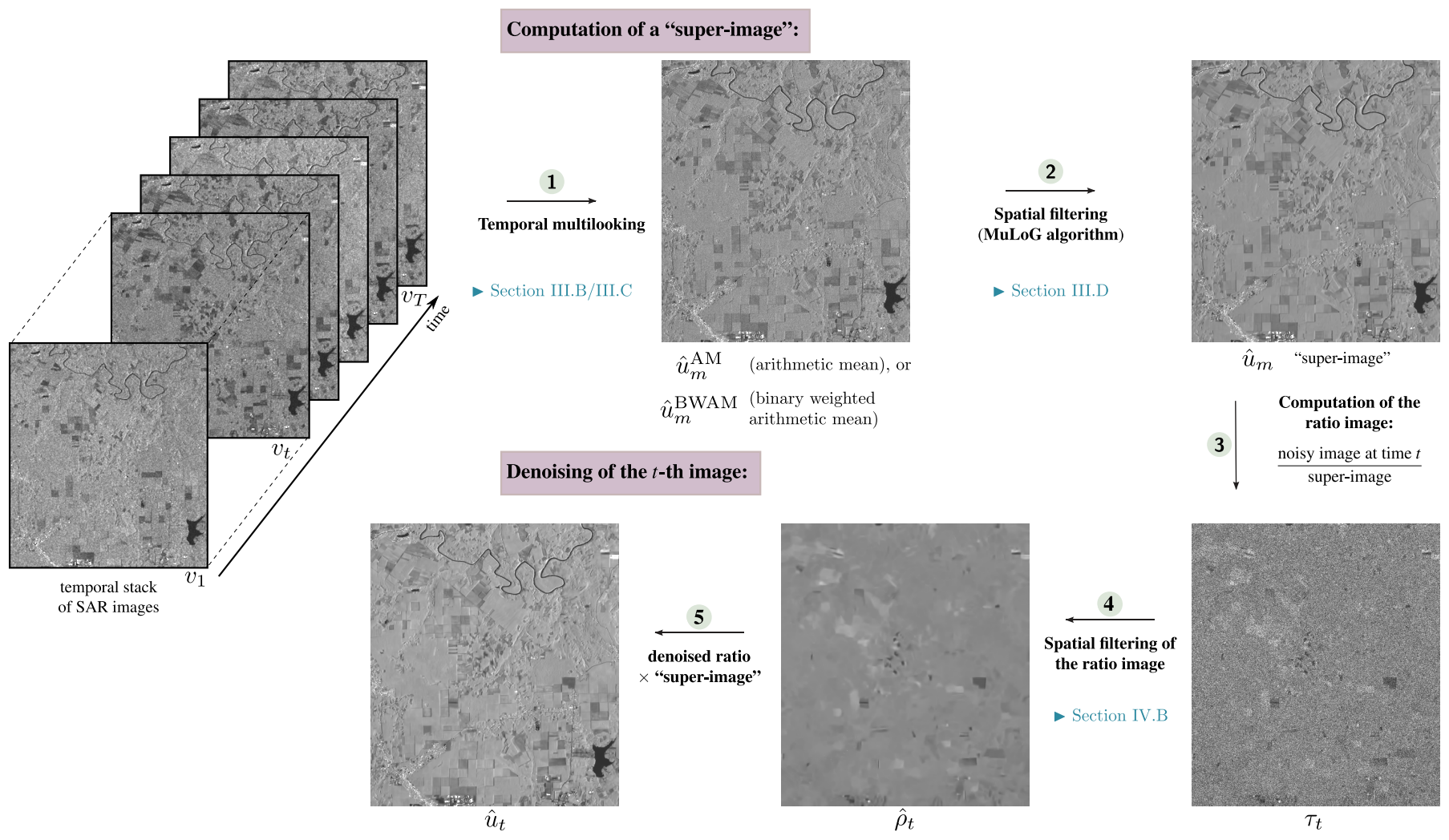

Fig. 1: Summary of RABASAR method for speckle reduction of SAR time series and associated sections of the paper.

arithmetic or the geometric means) could be chosen; then, these means may be applied either on the intensity data or on the amplitude data. We may expect from these choices to enhance different pieces of information [30]. In this paper, registered and radiometrically corrected intensity SAR images are used. We propose to use the arithmetic mean for its good properties [30], in particular in terms of modeling the statistics of the super-image, with the option of using binary weights to discard the intensity at some dates when a change occurred.

\section{A. Statistics of SAR images}

We briefly recall in this section the statistics of fully developed speckle. Under Goodman's hypothesis [31], fully developed intensity speckle follows a gamma distribution $\mathcal{G}(u, L)$ depending on the number of looks $L$ and the mean reflectivity $u$ (the aftersought signal of interest) of the scene:

$$
p_{v}(v \mid u, L)=\frac{L}{u \Gamma(L)}\left(\frac{L v}{u}\right)^{L-1} e^{-\frac{L v}{u}}
$$

Speckle in SAR images acts like a multiplicative noise and the speckle model can be expressed as [5]:

$$
v=u w
$$

where $w$ follows a gamma distribution $\mathcal{G}(1, L)$, the expectation is $\mathbb{E}[v]=u$ and the variance is $\operatorname{Var}[v]=u^{2} / L$. With the increase of the number of looks $L$, the variance decreases. The proportionality between the variance and $u^{2}$ reflects that noise is signal-dependent and multiplicative.

\section{B. Arithmetic mean}

Given a time series of $T$ intensity values $\left\{v_{t}(s)\right\}_{t=1}^{T}$ indexed by time $t$, the arithmetic mean is calculated at location $s$ by:

$$
\hat{u}_{m}^{\mathrm{AM}}(s)=\frac{1}{T} \sum_{t=1}^{T} v_{t}(s) \quad 1 \leq t \leq T
$$

Theoretically, with (i) no change in the time series, $u_{1}(s)=$ $u_{2}(s)=\cdots=u_{T}(s)$, and (ii) $T$ large enough and speckle sufficiently decorrelated from one image to an other, averaging the temporal intensity data is a simple yet effective approach to reduce the speckle [32]. When speckle is fully decorrelated, the arithmetic mean $\hat{u}_{m}^{\mathrm{AM}}(s)$ corresponds to the maximum likelihood estimation of $u(s)$ and the multi-look image $\hat{u}_{m}^{\mathrm{AM}}$ follows a gamma distribution $\mathcal{G}(u, L T)$. In practice, because of temporal correlations of the speckle, the resulting equivalent number of looks (ENL) may be less than this theoretical value $(L T)$, especially in case of images in interferometric configuration. The associated ENL will be denoted by $L_{m}$ in the following, and its estimation is discussed in Sec. III-D.

When changes occur in the time series, the arithmetic mean $\hat{u}_{m}^{\mathrm{AM}}(s)$ no longer matches the scene reflectivity at time $t$. We can reduce this discrepancy between the temporal average and the reflectivity at date $t$ by averaging only the unchanged temporal samples, as described in the next paragraph.

\section{Binary weighted arithmetic mean}

Instead of computing the super-image from the time series without considering the date $t$ of the image under study, 
another option is to compute a dedicated super-image that is closer to the reflectivity $u_{t}$. Only samples with similar and stable reflectivities can be considered in the average. To detect these samples, we suggest using a patch-based selection based on the generalized likelihood ratio (GLR) test. The GLR test between two independent observed intensity values $v_{1}$ and $v_{2}$ (with same number of looks $L$ ) confronts the following two hypotheses: the null hypothesis corresponding to a common reflectivity value $u_{12}$ accounting for the observed intensities $v_{1}$ and $v_{2}$, and the alternative hypothesis where a different reflectivity is considered for each observation $\left(u_{1}\right.$ for $v_{1}$ and $u_{2}$ for $v_{2}$ ) to account for a change in the reflectivity between the observations. The generalized likelihood ratio (GLR) test corresponding to this hypothesis test is given by [33]:

$$
\operatorname{GLR}\left(v_{1}, v_{2}\right)=\frac{v_{1} v_{2}}{\left(v_{1}+v_{2}\right)^{2}},
$$

where constant terms are omitted. Taking the log and extending the comparison to the image patches centered at location $s$ in the images at date $t$ and $t^{\prime}$ lead to [9], [24]:

$$
d_{t, t^{\prime}}(s)=\sum_{\delta} \log \left(\sqrt{\frac{v_{t}(s+\delta)}{v_{t^{\prime}}(s+\delta)}}+\sqrt{\frac{v_{t^{\prime}}(s+\delta)}{v_{t}(s+\delta)}}\right)
$$

where $v_{t}(s+\delta)$ is the value in the noisy patch at date $t$. The sum is taken over all pixel shifts $\delta$ such that pixels with index $s+\delta$ are located inside a patch centered on $s$ (small square window). Then, a binary weight $w_{t, t^{\prime}}(s)$, expressing whether there are temporal changes or not, is computed as:

$$
w_{t, t^{\prime}}(s)= \begin{cases}1, & \text { if } d_{t, t^{\prime}}(s)<\sigma \\ 0, & \text { otherwise }\end{cases}
$$

where $\sigma$ is a threshold chosen as $\sigma=$ quantile $\left(d_{t, t^{\prime}}(s), \alpha\right)$ under the null hypothesis and is estimated with Monte Carlo simulations using gamma distributed data and $\alpha=0.92$ as proposed in [24].

Then, the binary weighted arithmetic mean (denoted by BWAM in the sequel) is calculated at date $t$ by:

$$
\hat{u}_{m}^{\mathrm{BWAM}_{t}}(s)=\frac{1}{\sum_{t^{\prime}=1}^{T} w_{t, t^{\prime}}(s)} \sum_{t^{\prime}=1}^{T} w_{t, t^{\prime}}(s) v_{t^{\prime}}(s)
$$

where the notation $\mathrm{BWAM}_{t}$ is used to highlight that the superimage depends on the targeted date $t$. For each pixel $s$ and each date $t$ of the stack, the weights $w_{t, t^{\prime}}(s)$ select the dates $t^{\prime}$ for which no significant change occurred with respect to the image at date $t$. Thus, the binary weighted mean $\hat{u}_{m}^{\mathrm{BWAM}_{t}}(s)$ provides a more faithful estimation of $u_{t}(s)$. This improvement is obtained at the cost of an increased complexity since the superimage is date specific: the stack has to be processed for each date $t$, while a single super-image is computed once and for all with the (unweighted) arithmetic mean. The interest of using such a temporal mean will be evaluated in Sec. V.

In practice, the selection performed by the binary weights affects the associated ENL $L_{m}$ of the super-image. In the next paragraph, we describe how $L_{m}$ is estimated.

\section{ENL estimation}

The super-image may have a spatially varying ENL because of temporally changing areas, because of spatially-varying coherence, or because of the use of locally-computed binary weights. To robustly estimate $L_{m}$ in the super-image calculated with medium or high resolution temporal SAR images, the log-cumulant method [34] is used within sliding windows. Empirical expressions for the local first and second order logcumulant estimators are (for $N$ samples):

$$
\begin{aligned}
\hat{k}_{1}(s) & =\frac{1}{N} \sum_{\delta} \log \hat{u}_{m}(s+\delta) \\
\text { and } \quad \hat{k}_{2}(s) & =\frac{1}{N} \sum_{\delta}\left[\log \hat{u}_{m}(s+\delta)-\hat{k}_{1}(s)\right]^{2}
\end{aligned}
$$

The sums are computed over all pixel shifts $\delta$ such that pixels with index $s+\delta$ are located inside a square window with $N$-pixels centered on $s$ (in practice, we use $N=30 \times 30$ pixels). Assuming all samples in the local window are iid, we can obtain a local estimation of the ENL by inverting the following relationship (theoretical expression):

$$
\hat{k}_{2}(s)=\psi\left(1, \hat{L}_{m}(s)\right)
$$

where $\psi$ is the first-order Polygamma function [34]. Note that the traditional estimation method (by means of the ratio $\mathbb{E}\left[\hat{u}_{m}\right]^{2} / \operatorname{Var}\left[\hat{u}_{m}\right]$ ), the moment estimation method or the maximum likelihood (ML) estimation method could also be used for the ENL estimation [35].

Nevertheless, samples within local windows centered on $s$ are usually not iid, and the subsequent statistic $\hat{L}_{m}(s)$ underestimates $L_{m}(s)$. For this reason, once the ENLs have been locally estimated in all sliding windows, we consider a global ENL for the whole super-image obtained by $\hat{L}_{m}=$ quantile $\left(\hat{L}_{m}(s), \alpha\right)$ where $\alpha=0.98$ (almost the maximum). While it may be argued that the ENL varies spatially in the image, we found on several images obtained by different sensors that using a constant ENL value leads to satisfying results in the subsequent denoising steps.

\section{E. Denoising the super-image}

When the number of dates $T$ is not large enough or when the temporal correlation of speckle is too strong, the super-image obtained by (weighted) averaging suffers from significant remaining speckle fluctuations. A spatial filtering step is then necessary to improve the quality of the superimage $\hat{u}_{m}$. At this step, any speckle reduction method can be used. In this paper, we use MuLoG-BM3D [28] to perform this spatial filtering. MuLoG-BM3D will be discussed in more details in Sec. IV-B where we describe how it can be extended to process ratio images.

In summary, 4 super-images can be computed: the arithmetic mean image (according to Eq. (4)), the binary weighted arithmetic mean image (according to Eq. (8)), the denoised arithmetic mean image (DAM) and the denoised binary weighted arithmetic mean image (DBWAM). The impact of the method used to build the super-image on the output of RABASAR is evaluated in Sec. V. In the following, these four super-images will be indifferently denoted as $\hat{u}_{m}$. 


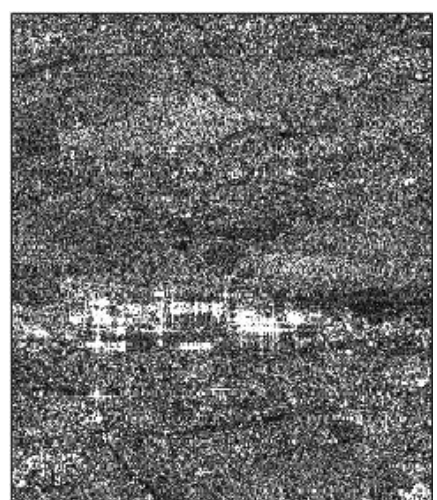

(a)

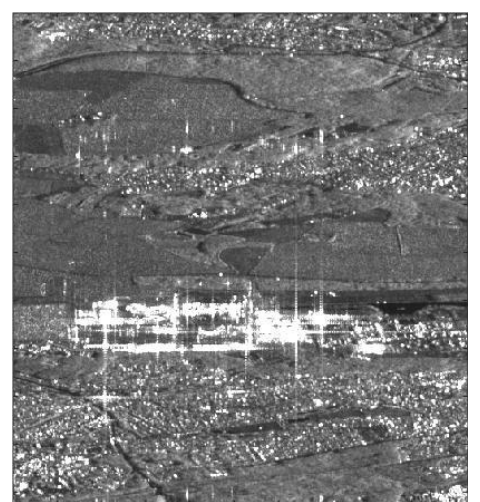

(b)

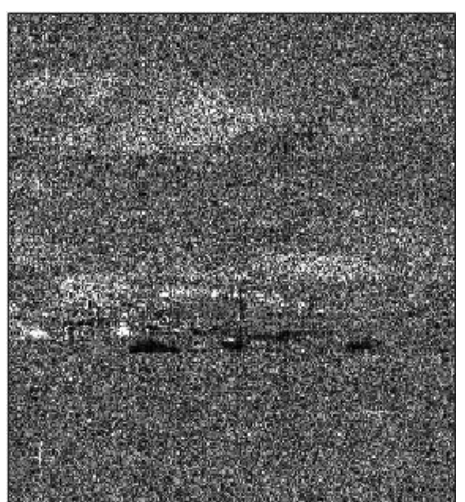

(c)

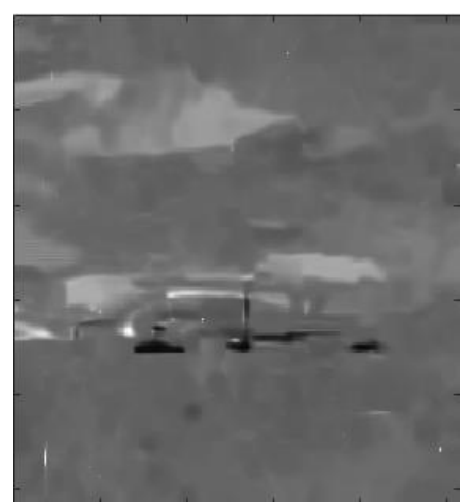

(d)

Fig. 2: (a) Sentinel-1 noisy image, (b) Arithmetic mean image, (c) Ratio image, (d) Denoised result on ratio image. Appearing (dark areas in (d)) and disappearing buildings (clear areas) are located in the middle of the image.

After temporal averaging and spatial filtering, the remaining noise in the super-image follows a distribution without known closed-form. It can nonetheless be approximated by a gamma distribution $\mathcal{G}\left(u_{m}, L_{m}\right)$ where $L_{m}$ is a constant parameter that can be re-estimated from the data in order to fit at best the empirical distribution as described in Sec. III-D.

\section{RATIO IMAGE DENOISING}

The ratio image $\tau_{t}$ formed at date $t$ contains both the fluctuations due to the speckle component of the noisy image $v_{t}$ (pure speckle) and some structures that appear due to the discrepancy between the super-image $\hat{u}_{m}$ and the reflectivity $u_{t}$ (changes of reflectivity). Compared to the noisy image $v_{t}$, the ratio image $\tau_{t}$ is spatially far more homogeneous so that denoising the ratio image is advantageous.

\section{A. Statistical analysis of the ratio}

Denoising a ratio image requires a denoising method adapted to the statistics of the ratio between the SAR image $v_{t}$ at date $t$ and the super-image $\hat{u}_{m}$. Despite the temporal averaging (and possibly, the spatial filtering) of the superimage, remaining fluctuations are present in $\hat{u}_{m}$. The statistical distribution of the ratio $\tau_{t}=v_{t} / \hat{u}_{m}$ therefore differs from the gamma distribution $\mathcal{G}\left(u_{t} / u_{m}, L\right)$ followed by the more ideal ratio $v_{t} / u_{m}$. Note that, for the sake of notation simplicity, we drop in this section the dependency with $s$ and each formula must be understood pixelwise. The Mellin framework developed in [35] provides an efficient way to derive the pdf of $\tau_{t}$. Indeed, the pdf of the product of two independent random variables is the Mellin convolution of the pdf of the two variables. The details of the computation are given in Appendix. It is shown that the ratio $\tau_{t}=v_{t} / \hat{u}_{m}$ follows a Fisher pdf $\mathcal{F}\left(\rho_{t}, L, L_{m}\right)$ with the three parameters $\rho_{t}=u_{t} / u_{m}, L$ and $L_{m}$ :

$$
p_{\tau}\left(\tau_{t} \mid \rho_{t}, L, L_{m}\right) \propto \frac{\tau_{t}^{L-1}}{\rho_{t}^{L}} \cdot\left(L_{m}+L \frac{\tau_{t}}{\rho_{t}}\right)^{-L-L_{m}}
$$

where the normalization constant depends solely on the number of looks $L$ and $L_{m}$. Fisher random variables have a multiplicative behavior, $\tau_{t}=\rho_{t} w_{t}$, where $w_{t}$ follows a Fisher distribution $\mathcal{F}\left(1, L, L_{m}\right)$. We have $\mathbb{E}\left[\tau_{t}\right]=\rho_{t} \frac{L_{m}}{L_{m}-1}$, for
$L_{m}>1$, and $\operatorname{Var}\left[\tau_{t}\right]=\rho_{t}^{2} \frac{L_{m}^{2}\left(L_{m}+L-1\right)}{L\left(L_{m}-2\right)\left(L_{m}-1\right)^{2}}$, for $L_{m}>2$. The proportionality between $\operatorname{Var}\left[\tau_{t}\right]$ and $\rho_{t}^{2}$ reveals again that noise is signal-dependent.

\section{B. Denoising of the ratio image: RuLoG algorithm}

To denoise the ratio images, we need a method that can account for the Fisher distribution that arises when forming the ratio between gamma-distributed random variables with differing number of looks. To this end, we describe in this paragraph the MuLoG framework [28] and how it can be extended in order to apply general-purpose Gaussian denoisers to Fisher-distributed noise.

MuLoG first stabilizes the variance by applying a logarithm transform to the image. We denote by $y$ the log of the ratio image $\tau_{t}$ at date $t$ (the dependency to $t$ is dropped to simplify the notations) and by $x=\log \rho_{t}=\log u_{t} / u_{m}$ the $\log$ of the ratio of the reflectivities. Thanks to the log transform, the multiplicative noise in the ratio image is mapped to an additive signal-independent noise with expectation $\mathbb{E}[y]=x-\log \frac{L}{L_{m}}+$ $\Psi(L)-\Psi\left(L_{m}\right)$ and variance $\operatorname{Var}[y]=\psi(1, L)+\psi\left(1, L_{m}\right)$ where $\Psi(\cdot)$ denotes the digamma function. This shows that, like for gamma-distributed noise, taking the log of the ratio image stabilizes the variance of the Fisher-distributed noise.

Unlike standard homomorphic approaches, and inspired by [36], MuLoG considers next the exact distribution of logtransformed data. Specifically, the $n$-pixels image $x$ is obtained by maximum a posteriori estimation expressed as the solution of an optimization problem of the form:

$$
\hat{x} \in \underset{x \in \mathbb{R}^{n}}{\arg \min }\left[-\log p_{y}(y \mid x)+\mathcal{R}(x)\right]
$$

where the first term $-\log p_{y}(y \mid x)$ is the exact likelihood of the log-transformed ratio $y$, and the second term $\mathcal{R}(x)=$ $-\log p_{x}(x)$ is a prior enforcing some spatial regularity on the solution. MuLoG solves Problem (13) iteratively by the alternating direction method of multipliers (ADMM) algorithm 
that repeats, for $\beta>0$, the updates

$$
\begin{aligned}
& \hat{z} \leftarrow \underset{z \in \mathbb{R}^{n}}{\arg \min }\left[\frac{\beta}{2}\|z-\hat{x}+\hat{d}\|^{2}+\mathcal{R}(z)\right] \\
& \hat{d} \leftarrow \hat{d}+\hat{z}-\hat{x} \\
& \hat{x} \leftarrow \underset{x \in \mathbb{R}^{n}}{\arg \min }\left[\frac{\beta}{2}\|x-\hat{z}-\hat{d}\|^{2}-\log p_{y}(y \mid x)\right]
\end{aligned}
$$

In practice, six iterations are considered with $\beta=1+2 / L+$ $2 / L_{m}$ as suggested in [28].

Regarding Problem (16), in the case of gamma-distributed speckle considered in the original MuLoG method [28], the likelihood term was corresponding to a Fisher-Tippett distribution. As discussed in the previous section, the distribution of ratio-images is not gamma-distributed but follows a Fisher distribution. Therefore the optimization of (16) has to be modified compared to the original MuLoG algorithm. By applying a change of variable, it can be shown that, after the $\log$ transform, Fisher-distributed random variables follow a $z$ Fisher pdf [37] given by:

$$
\begin{array}{r}
p_{r}\left(y(s) \mid x(s), L, L_{m}\right) \propto \exp [L(y(s)-x(s))] \\
\cdot\left(L_{m}+L \exp [y(s)-x(s)]\right)^{-L-L_{m}}
\end{array}
$$

which leads to:

$$
\begin{aligned}
& -\log p_{y}(y \mid x)=\mathrm{Cst}+\sum_{s=1}^{N}[L x(s) \\
& \left.+\left(L+L_{m}\right) \log \left(L_{m}+L \exp [y(s)-x(s)]\right)\right] .
\end{aligned}
$$

Injecting this expression in (16) leads to solve $N$ separable convex problems whose solutions can be obtained by a few iterations of Newton's method defined by:

$$
\hat{x}(s) \leftarrow \hat{x}(s)-\frac{\beta(\hat{x}(s)-\hat{z}(s)-\hat{d}(s))+L(1-c(s))}{\beta+L c(s)\left(1-\frac{L}{L_{m}+L} c(s)\right)}
$$

where $c(s)=\left(L_{m}+L\right) \exp [y(s)-\hat{x}(s)] /\left\{L_{m}+L \exp [y(s)-\right.$ $\hat{x}(s)]\}$. Interestingly, when $L_{m} \rightarrow \infty$, the z-Fisher pdf tends to the Fisher-Tippet pdf, and taking the limit in (19) leads to the original MuLoG algorithm [28].

Regarding Problem (14), MuLoG adopts the strategy of plug-and-play ADMM (see for instance [38]) which consists in replacing the minimization problem involving $\mathcal{R}(x)$ by the solution of a denoiser adapted to additive white Gaussian noise with variance $1 / \beta$. In this paper, we consider using BM3D [39], an algorithm based on patch similarity and three-dimensional wavelet shrinkage, and reaching remarkable results with fast computation. We refer to this method for ratio image denoising as Ratio adaptation of MuLoG (RuLoG), see Algorithm 1.

After obtaining the estimated noise-free value $\hat{\rho}_{t}(s)=$ $\exp (\hat{x}(s))$ of the ratio, we obtain the denoised image $\hat{u}_{t}$ through:

$$
\hat{u}_{t}(s)=\hat{u}_{m}(s) \cdot \hat{\rho}_{t}(s)
$$

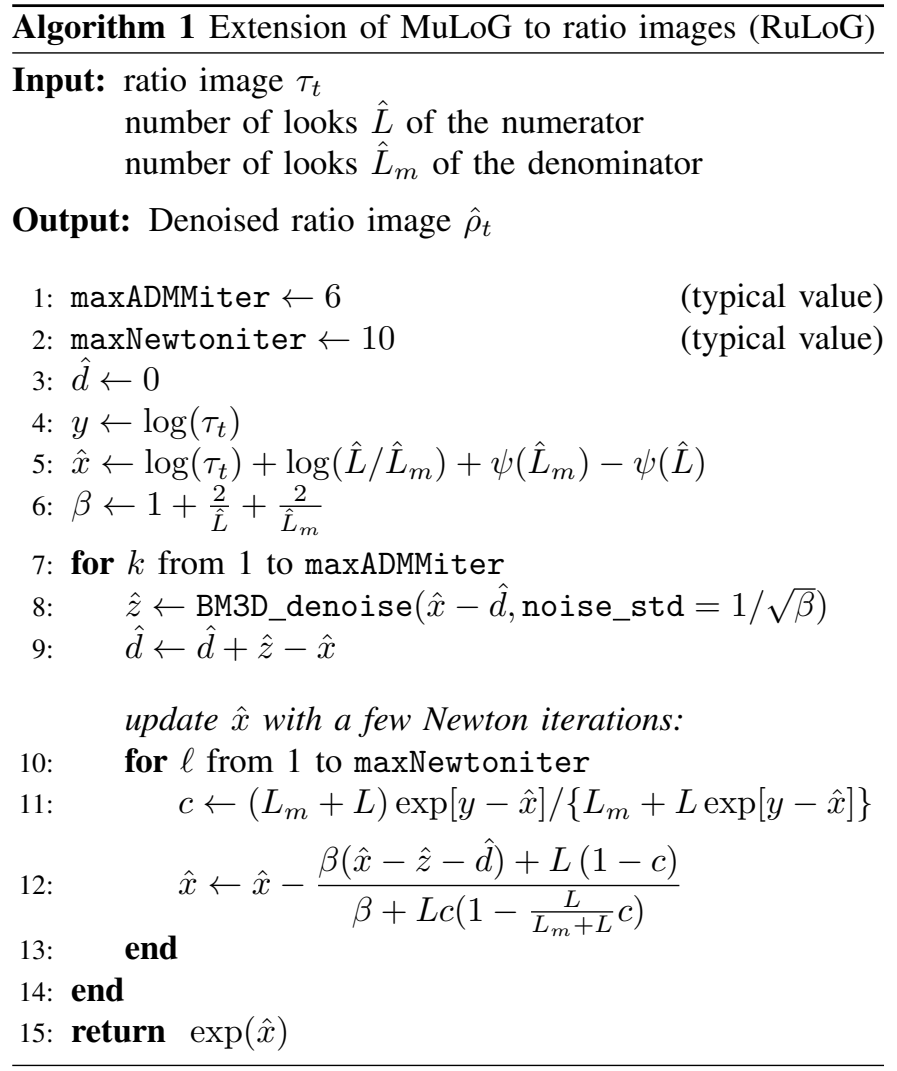

$\overline{\text { Algorithm } 2 \text { Multi-temporal speckle reduction (RABASAR) }}$

Input: $T$ co-registered SAR images $\left\{v_{t}\right\}_{t=1}^{T}$, targeted date $t$, input number of looks $L$.

Output: Image with reduced speckle $\hat{u}_{t}$.

Step 1: computation of the super-image (AM or BWAM)

1: $\hat{u}_{m} \leftarrow$ compute_super_image $(y, t)$

2: $\hat{L}_{m} \leftarrow$ estimate_looks $\left(\hat{u}_{m}\right)$

Step 2 (optional): denoising of the super-image

3: $\hat{u}_{m} \leftarrow$ MuLoG_BM3D $\left(\hat{u}_{m}, L=\hat{L}_{m}\right)$

4: $\hat{L}_{m} \leftarrow$ estimate_looks $\left(\hat{u}_{m}\right)$

Step 3-4: denoising of the ratio image

5: $\tau_{t} \leftarrow v_{t} / \hat{u}_{m}$

6: $\hat{\rho}_{t} \leftarrow$ RuLoG_BM3D $\left(\tau_{t}, L, L_{m}=\hat{L}_{m}\right)$

Step 5: computation of the restored image

7: $\hat{u}_{t} \leftarrow \hat{u}_{m} \cdot \hat{\rho}_{t}$

8: return $\hat{u}_{t}$

The application of this pipeline - RABASAR - is illustrated Fig. 2. A typical filtering result on a ratio image is presented on Fig. 2(d) along with the original image, super-image and the ratio image. The pseudo-code is given in Algorithm 2.

\section{EXPERIMENTAL RESULTS}

To evaluate the performances of the proposed method, different experiments have been conducted on simulated 


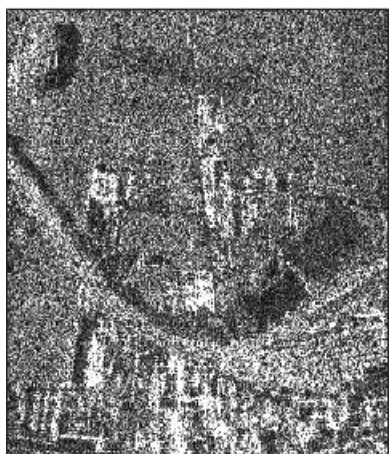

(a)

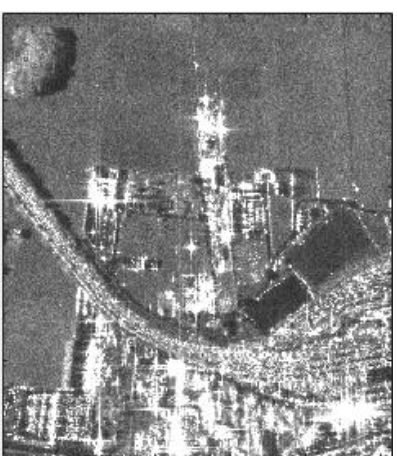

(b)
Fig. 3: TerraSAR-X series: (a) one image of the temporal series, (b) arithmetic mean of the 26 images.

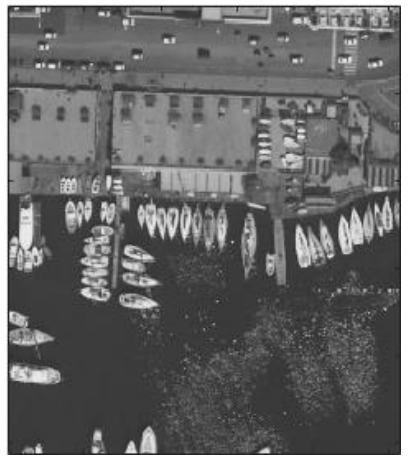

(a)

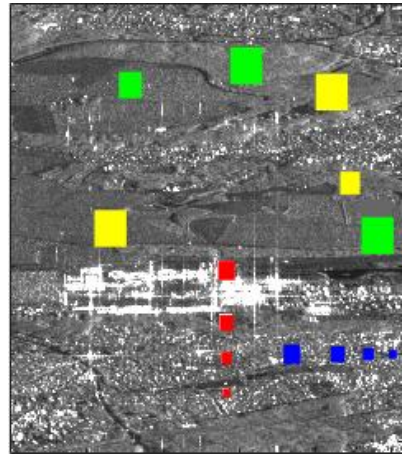

(b)
Fig. 4: Images used for data simulation (a) optical image used as a noise free image, (b) arithmetic mean of Sentinel-1 images with the location of different land-cover types used to simulate temporal changes (green: farmland changes, yellow: forest changes, red: appearing building, blue: appearing then disappearing building.

and SAR images. Recall that an open-source code of RABASAR is available at https://www.math.u-bordeaux.fr/ $\sim$ cdeledal/rabasar.php.

The influence of different super-images on RABASAR denoising results are commented in Sec. V-C. Then, RABASAR is compared to some selected methods from the literature: UTA [17], [18], NLTF[21], 2SPPB [24] and MSAR-BM3D [21] (Sec. V-D).

\section{A. Data presentation}

1) Sentinel-1 and TerraSAR-X images: The proposed methods are tested on a time series of 69 descending Sentinel-1 IW SLC Level-1 images acquired from December 24, 2014 to May 6, 2017 with VV polarization over Saclay area, south of Paris ${ }^{1}$ (see Fig. 2(a) and (b)). Saclay plateau is mostly an agricultural area with pieces of forests and dispersed academic buildings. In the last five years, many new buildings and infrastructures have been under development.

In addition, 26 single-look TerraSAR-X images (13 images are sensed in 2009 and the other 13 images in 2011) acquired over Saint-Gervais-les-Bains, south-east of Geneva, are

${ }^{1}$ All the Sentinel-1 images can be downloaded from Copernicus Open Access Hub (https://scihub.copernicus.eu/dhus/). used (project DLR-MTH0232). These images are taken over a highly mountainous countryside, with a narrow inhabited valley concentrating many human artifacts (roads, bridges, dams) (see Fig.3).

In this paper we are dealing with images acquired in interferometric configuration (same orbit and incidence angle). In this case, the registration step can be done accurately using the sensor parameters provided by the space agencies. Besides Sentinel-1 and TerraSAR-X images are radiometrically calibrated.

It often happens that SAR pixels are not spatially independent because of a slight over-sampling creating a spatial correlation. This spatial correlation should be taken into account during the denoising. However, most of the denoising methods are based on the hypothesis that the speckle noise is white. When applied directly to images with correlated noise, reduced performances may be expected from these methods. Therefore, we recommend to perform a spatial decorrelation before despeckling. In this paper, the noisy TerraSAR-X images are decorrelated using the method proposed in [40] and the Sentinel-1 images are decorrelated by resampling because of its special acquisition model (the beam both steering in range direction and steering from backward to forward in azimuth direction). All the SAR images are co-registered using subpixel image registration applied on the single look complex data [27].

2) Simulated data: Simulated SAR images are obtained according to Eq. (3), by multiplying a reflectivity map with a random gamma distributed noise.

Many simulations are based on reflectivity maps obtained from optical images. However, SAR images exhibit strong and persistent scatterers, especially in urban areas which can hardly be simulated using optical images. Therefore, we propose to use the arithmetic mean image of long time-series of SAR images, considered as a noise free image (a reflectivity map $u$ ) to create realistic simulations of SAR images. This map $u$ is multiplied by a gamma distributed noise $w_{t}$ providing an image $v_{t}=u w_{t}$ of the series. These simulated data correspond to the case without change and are an ideal case (pure iid samples of noise). Images of Fig.2(b), Fig.3(b) and 4(a) are used for the simulated case without changes.

Concerning changes in the temporal series, to deal with changes in a realistic way, temporal sequences have been introduced over various areas: forests, farmlands, building areas, etc. as shown in Fig.4(b). The introduced temporal changes have been chosen according to changes observed in SAR time series and have the temporal profiles shown in Fig. 6.

\section{B. Evaluation method}

Measurement of speckle reduction performances is a challenging task, especially when noise-free data are unavailable. Visually checking the despeckling results is an immediate and important way for quality evaluation, but it lacks objectivity. To overcome this limitation, we use the peak signal-noiseratio (PSNR) and structure similarity (SSIM) indexes, even though they suffer from some limitations that have been well discussed in the literature. 


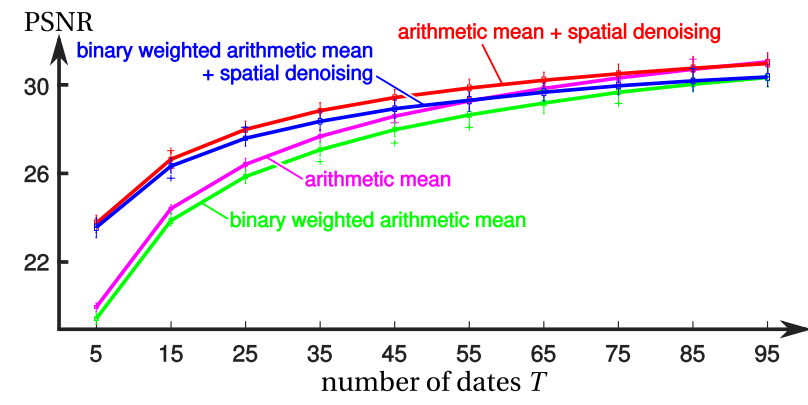

(a)

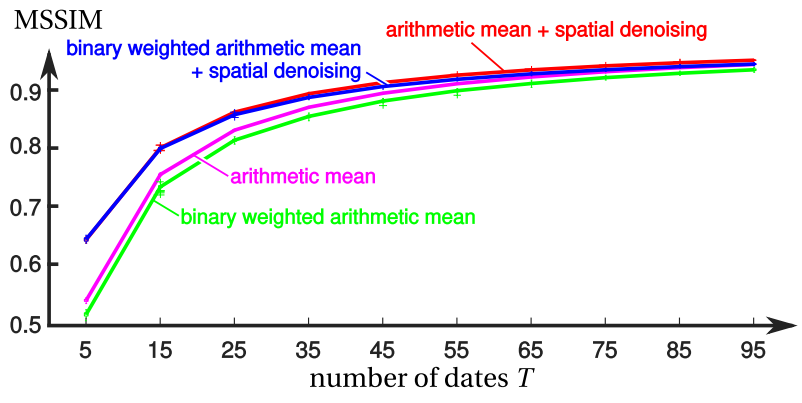

(b)

Fig. 5: RABASAR performances obtained with different super-images, in the absence of change, as measured with the PSNR (a) and the MSSIM (b). The solid line represent the mean value computed over 50 different noise realizations. For each index box, the bottom and top edges indicate the 25 th and 75 th percentiles, respectively. The outliers are plotted individually using the '+' symbol.

1) PSNR: Peak signal-noise-ratio: The PSNR is a commonly used approach to evaluate the quality of restoration results. We consider the PSNR expressed on amplitude images $u_{A}=\sqrt{u}$ and $\hat{u}_{A}=\sqrt{\hat{u}}$ :

$$
\operatorname{PSNR}=10 \cdot \log _{10} \frac{\left|u_{A}\right|_{\max }^{2}}{\mathbb{E}\left[\left(u_{A}(s)-\hat{u}_{A}(s)\right)^{2}\right]}
$$

where $\left|u_{A}\right|_{\max }$ is the maximum amplitude value in the noise free data, $\mathbb{E}[\cdot]$ represents the spatial average and $\hat{u}_{A}$ is the denoised amplitude value.

2) MSSIM: Mean structure similarity index: To evaluate the preservation of image features, the SSIM index [41] (structural similarity index measurement) is often preferred to PSNR. From the SSIM, we derive the mean structural similarity index measurement (MSSIM) values which provide a comprehensive measure over the whole image:

$$
\begin{aligned}
\operatorname{MSSIM}=\frac{1}{N} \sum_{i=1}^{N}\left[\frac{2 \cdot \widehat{\mathbb{E}}\left[u_{A}\right] \cdot \widehat{\mathbb{E}}\left[\hat{u}_{A}\right]+\alpha_{1}}{\widehat{\mathbb{E}}\left[u_{A}^{2}\right]+\widehat{\mathbb{E}}\left[\hat{u}_{A}^{2}\right]+\alpha_{1}}\right. \\
\frac{2 \cdot \widehat{\operatorname{Cov}}\left[u_{A}, \hat{u}_{A}\right]+\alpha_{2}}{\left.\widehat{\operatorname{Var}}\left[u_{A}\right]+\widehat{\operatorname{Var}}\left[\hat{u}_{A}\right]+\alpha_{2}\right]}
\end{aligned}
$$

where $u_{A}$ and $\hat{u}_{A}$ are noise free and denoised amplitude patches, $\operatorname{Cov}[\cdot, \cdot]$ is the measure of covariance, $\alpha_{1}$ and $\alpha_{2}$ are suitable constants, and $N$ is the number of local windows in the image.

\section{Which super-image gives the best denoising?}

We presented in Sec. III-E different ways to compute the super-image (AM, BWAM and their spatially denoised versions). This section presents quantitative (Fig. 5) and qualitative (Fig. 7) denoising results obtained when using different super-images, and considering different time series lengths $T$.

In the binary weight computation (methods BWAM and DBWAM), a window of size $7 \times 7$ is used in Eq. (6), as suggested by the analysis in [42].

1) Using simulated radar images: Simulated images allow to evaluate quantitatively the performances obtained with each of the four different super-images by measuring averaged PSNR and MSSIM values (50 different noise realization are computed in order to compute the average PSNR and MSSIM values). The number of images $T$ in the time series varies from 5 to 95 .

For temporal images without reflectivity changes (Fig. 5(ab)), using denoised super-images provides better PSNR and MSSIM values when using a small number of images, but this benefit disappears with longer stacks of images (more than 60). When using denoised super-images, the obtained PSNR/MSSIM values is notably impacted by the value of the ENL.

In the case of changing areas, we performed restorations of 50 speckle realizations in the four different change scenarios discussed in paragraph V-A2. We report in Fig. 6 the mean value and $1 \sigma$ confidence interval obtained by RABASAR. It can be observed that, in the case of fluctuations typically observed in farmland and forest areas (Fig. 6 (a) and (b)), both RABASAR-AM and RABASAR-BWAM perform well and produce an estimate with no significant bias (the reflectivity varies up to a factor 2 in our simulations). Because a smaller number of dates is selected in the case of RABASAR-BWAM, the estimation variance is very slightly larger. In Fig. 6 (c) and (d) we consider the case of much larger changes that typically occur in urban areas: an appearing large reflectivity or a temporarily large reflectivity. In this case, RABASAR-AM leads to a bias of the same order as the estimation standard deviation. The origin of this bias, observed in Fig. 6(c) for $t \geq 29$, can be ascribed to the difference between the true reflectivity and the mean intensity. This difference is too small to be compensated when denoising the ratio. When $t<29$, the difference is much larger and no bias can be observed (after denoising, the ratio image correctly compensates for the misfit of the super-image value). By selecting only similar dates, RABASAR-BWAM is more robust to this phenomenon: the bias is negligible compared to the estimation standard deviation. In the case of an appearing, then disappearing structure (Fig. 6(d)), no significant bias is visible with either method because the super-image with RABASAR-AM differs significantly from the true reflectivity and that difference is correctly recovered when denoising the ratio image (the signalto-noise ratio is sufficient so that it be recovered).

2) Using Sentinel-1 images: The temporal series of 69 Sentinel-1 images on the Saclay area is used to test the method 
(a)

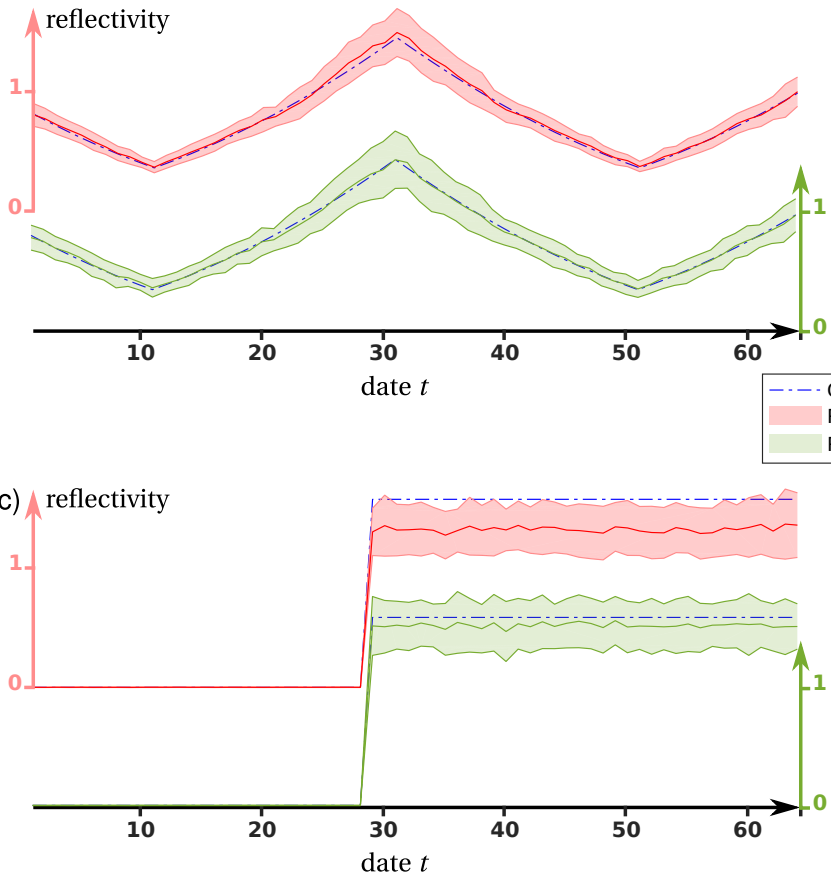

(b)

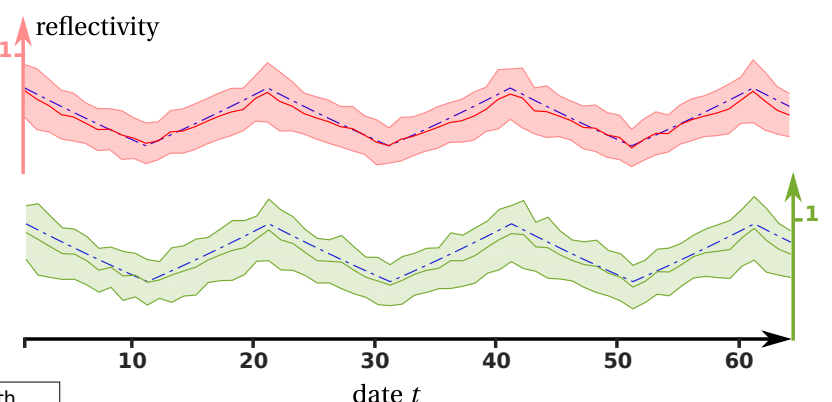

RABASAR-AM

RABASAR-BWAM

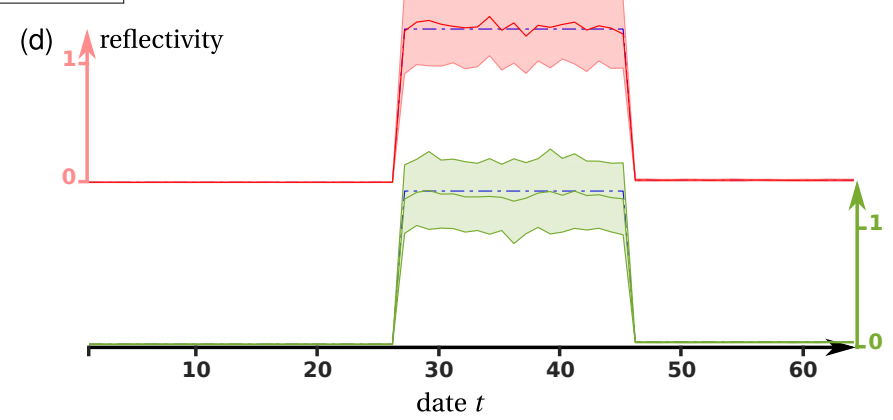

Fig. 6: Evaluation of RABASAR estimators in 4 different change scenarios: typical temporal variations of reflectivities in (a) farmland areas; (b) forest areas; (c) urban areas when a new building appears; (d) urban areas with an appearing then disappearing structure. The mean and $1 \sigma$ confidence interval are displayed for RABASAR-AM (top curve) and RABASARBWAM (bottom curve).

on SAR images. Figure 7 can be used to visually assess the efficiency of speckle reduction when using different "superimages". RABASAR provides satisfying denoising results with each of the four different super-images. The use of an additional spatial filtering step to form the super-image seems beneficial in terms of restoration quality: the obtained images are smoother.

When using AM and DAM, small areas with low values were sometimes smoothed, leading to the apparition of new points in the denoised results (Fig.7(a-b) red rectangular). This phenomenon is obvious for impulsive and abrupt changes in building areas. Using BWAM and DBWAM reduces this problem (Fig. 7(c-d)). In some changing parts of the image, using BWAM, however, leads to poor filtering results because very few similar dates could be combined when computing the super-image.

3) Computation time: The computation time of the algorithm depends on the adopted RABASAR version (type of super-image -binary weighted or not-, ENL estimation method and choice of the spatial denoising).

When running the experiments on a time serie of 69 SAR images of size $512 \times 768$ (Saclay) in the MATLAB environment on a computer (4 cores, Intel(R) Core(TM) i7-7600U CPU @ 2.80GHz), the averaged elapsed time of RABASARDBWAM is given in Table I.

As can bee seen, the main part of RABASAR computing time is due to the denoising step. If both the super-image and the ratio image are denoised, the time is multiplied by 2 . The similarity and weight computation represents only $1 \%$ of the total time. Nevertheless, when using binary weighted
TABLE I: AVERAGED COMPUTATION TIME OVER TEN RUNS OF THE DIFFERENT STEPS FOR A TIME SERIE OF 69 IMAGES WITH SIZE $512 \times 768$ PIXELS

\begin{tabular}{lllr}
\hline Step & & \multicolumn{3}{c}{ Averaged time } \\
\hline \hline BWAM super-image & (step 1) & $0.74 \mathrm{sec}$ & $(1.0 \%)$ \\
ENL estimation & (step 1 and 2) & $0.18 \mathrm{sec}$ & $(0.3 \%)$ \\
MuLog-BM3D & $($ step 2) & $34.3 \mathrm{sec}$ & $(50.5 \%)$ \\
RuLog-BM3D & $($ step 4) & $32.7 \mathrm{sec}$ & $(48.2 \%)$ \\
$\quad \rightarrow$ ADMM itself & $($ step 2 \& 4) & $1.90 \mathrm{sec}$ & $(2.8 \%)$ \\
$\quad \rightarrow$ BM3D itself & $($ step 2 \& 4) & $65.1 \mathrm{sec}$ & $(95.9 \%)$ \\
\hline Total time & & $67.9 \mathrm{sec}$. & \\
\hline
\end{tabular}

arithmetic mean, the super-image has to be computed again for each image to process, whereas the super-image computation is done only once for AM or DAM versions of RABASAR.

\section{Denoising performances of RABASAR compared to exist- ing methods}

The proposed method is compared with state-of-the-art multi-temporal denoising methods, both on simulated and SAR images. Numerical and visual results are provided when comparing RABASAR with the chosen methods: UTA [18], NLTF [21], 2SPPB [24] and MSAR-BM3D [21].

1) Quantitative comparison: As in the previous sections, to quantitatively compare the filtering performance of these methods, averaged PSNR and MSSIM are computed. Only RABASAR-AM and RABASAR-DAM performances are presented in the curves of Fig. 8.

In this simulation, we see from Fig. 8, that RABASAR generally provides better PSNR than other filtering methods. 


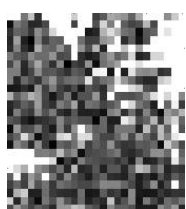

Noisy

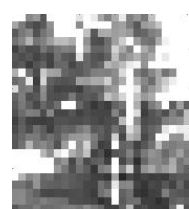

$\mathrm{AM}$

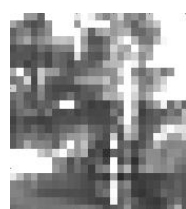

DAM

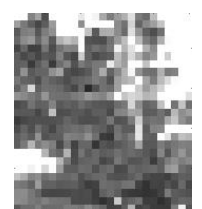

BWAM

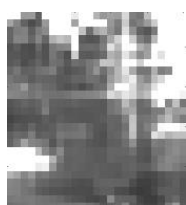

DBWAM
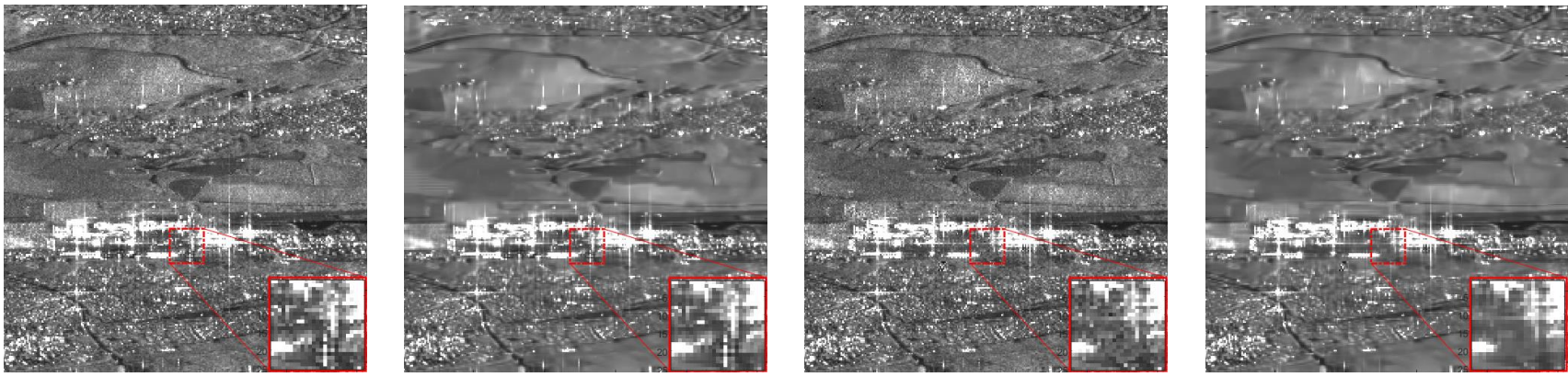

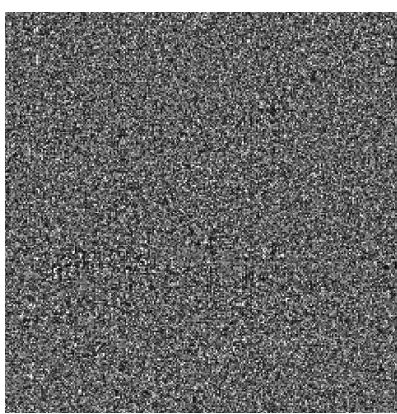

(a) RABASAR-AM

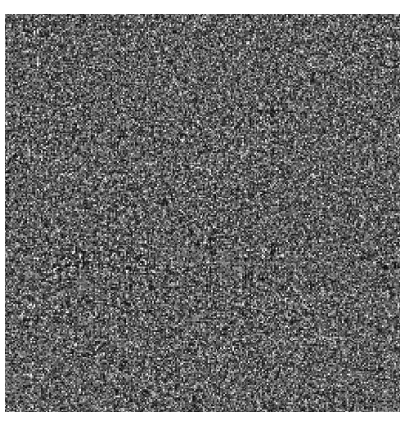

(b) RABASAR-DAM

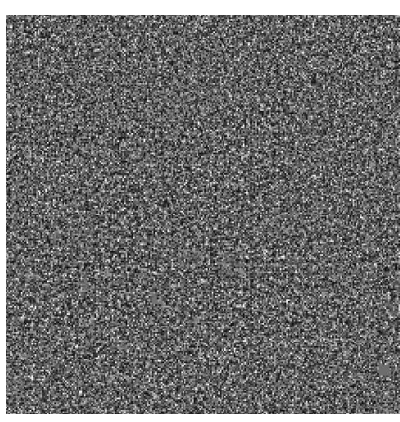

(c) RABASAR-BWAM

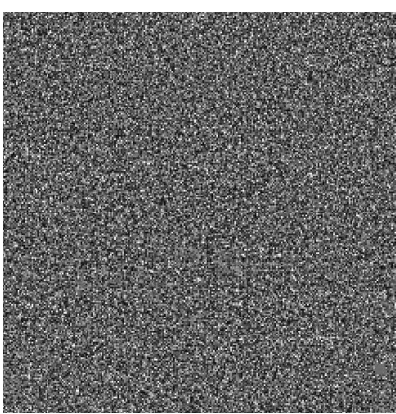

(d) RABASAR-DBWAM

Fig. 7: RABASAR denoising results on Sentinel-1 images over the Saclay area (middle row) and corresponding ratio-images (lower row) based on the use of: (a) AM, (b) DAM, (c) BWAM, (d) DBWAM. 69 Sentinel-1 images are used. The zoom of the red box is enlarged in the first row together with the original noisy image to ease the comparison.

TABLE II: NUMERICAL RESULTS PROVIDED BY DIFFERENT METHODS WITH 32 IMAGES IN THE SERIES. EVALUATION IS MADE BY PSNR AND MSSIM. FOR PSNR AND MSSIM, LARGER VALUES EXPRESS BETTER DENOISING RESULTS. THE MEAN VALUES OF 20 TIMES TEST ARE USED.

\begin{tabular}{c|c|cccc|cccc}
\hline Sentinel-1 & Evaluation & UTA & NLTF & 2SPPB & MSAR & \multicolumn{4}{c}{ RABASAR } \\
& methods & & & & -BM3D & -AM & -DAM & -BWAM & -DBWAM \\
\hline without & PSNR & 25.37 & 19.49 & 25.16 & 21.90 & 27.34 & $\mathbf{2 8 . 6 1}$ & 26.72 & 28.15 \\
changes & MSSIM & 0.84 & 0.82 & 0.83 & 0.67 & 0.86 & $\mathbf{0 . 8 9}$ & 0.85 & 0.88 \\
\hline
\end{tabular}

With few images in the stack, MSAR-BM3D and 2SPPB also provide competitive PSNR and MSSIM. However, with the increase of the number of images, MSAR-BM3D curve does not rise as fast as 2SPPB. When using less than 4 images, MSAR-BM3D provides the best MSSIM values. With an increasing number of images, UTA PSNR and MSSIM values keep increasing.

Table II presents some results about PSNR and MSSIM results. It shows that RABASAR-DAM provides the best results in this ideal situation when there is no change in the temporal series.

2) Sentinel-1 and TerraSAR-X image denoising: This section presents and discusses the results obtained when denoising Sentinel-1 and TerraSAR-X images. Since the noise free images are not available, the denoised results and the residual noise (ratio of the original image by the denoised image) are visually evaluated. The residual noise should correspond to pure gamma-distributed noise samples of mean 1. Residual structures, homogeneous areas, and radiometric variations correspond to the following perturbations: the destruction of structures, the absence of filtering, and bias introduction.

First, compared to MuLoG-BM3D applied on a single image, RABASAR-DAM provides a much better result, preserving fine strucures and isolated objects, see Fig. 11. This experiment shows that the exploitation of the super-image and the ratio image, which is much more stationary than the original noisy image, helps preserving the original resolution.

Figure 9 presents different filtering results on a Sentinel1 time serie of Saclay. Compared to 2SPPB and MSARBM3D methods, RABASAR-DAM and RABASAR-DBWAM 

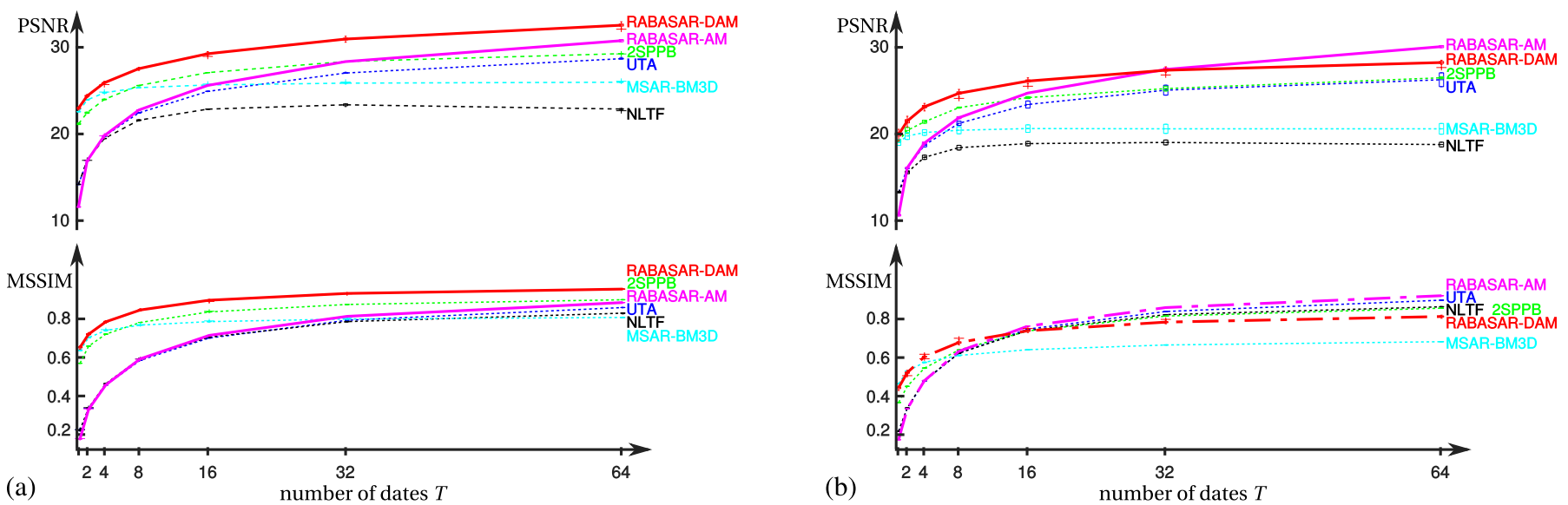

Fig. 8: Comparison of different temporal denoising methods: UTA, NLTF, 2SPPB, MSAR-BM3D, RABASAR-AM and RABASAR-DAM. Averaged PSNR (upper row) and MSSIM (lower row) as a function of the number of images for two data sets: (a) simulated time serie without changes using Fig.4(a)), (b) realistic simulated SAR data without changes using Fig. 4(b)). Each experiment is repeated 50 times, with the same noise free value multiplied by different gamma distribution noise. The line features shows the mean value of PSNR (top) and MSSIM (bottom)over the 50 experiments.

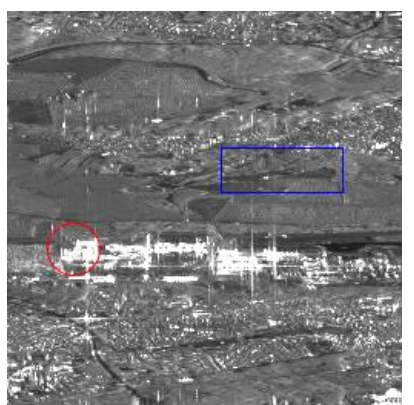

(a)

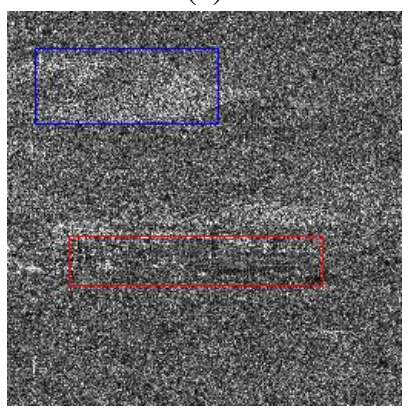

(e)

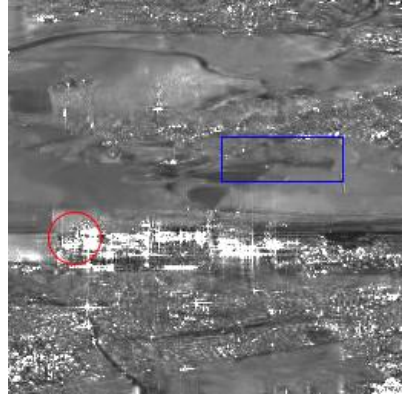

(b)

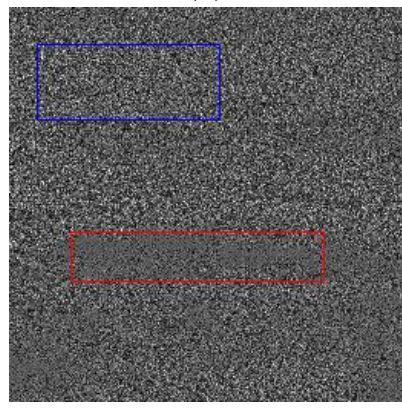

(f)

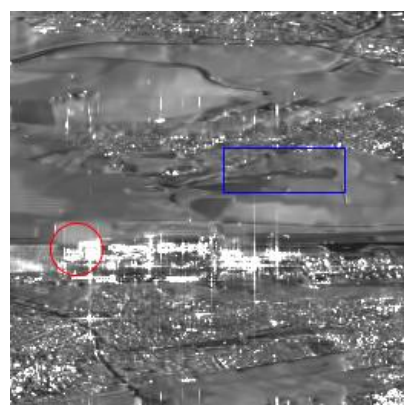

(c)

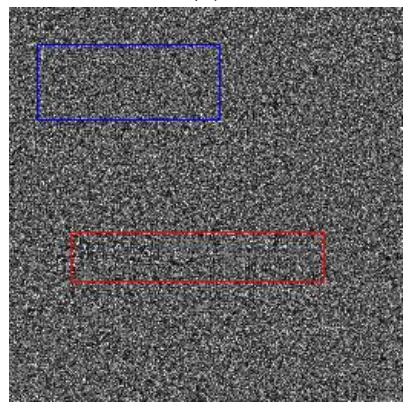

(g)

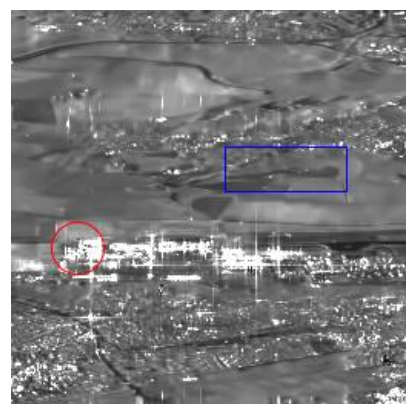

(d)

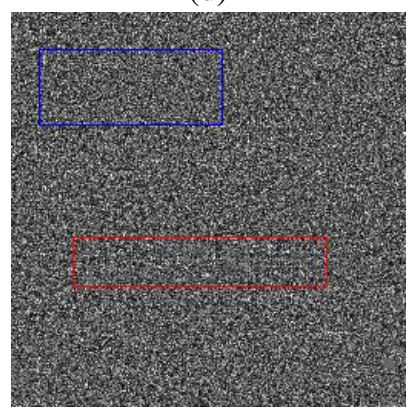

(h)

Fig. 9: Denoising Sentinel-1 images over the region of Saclay (RuLoG results) (the original noisy image is shown in Fig. 2(a)): (a) filtered with 2SPPB, (b) with MSAR-BM3D, (c) with RABASAR-DAM, (d) with RABASAR-DBWAM, (e-h) residual noise images between the noisy image and denoised results. 69 Sentinel-1 images are used.

provide better denoising results since they take both the ratio results and the texture characteristics into account. Some noisy areas can be observed in the RABASAR-DBWAM results due to the temporal samples selection (for some pixels, only few similar temporal samples can be found to compute the binary weighted arithmetic mean). 2SPPB method does not give good results for seasonal changing farmland areas, and shows an obvious bias in the residual noise (see for instance the blue circle area in Fig. 9(e)).

Since MSAR-BM3D method detects the bright points in advance and prohibits any denoising around these points [21], building areas in the residual noise are homogeneous (Fig. 9(f) red circle area) showing that no filtering has been applied in these areas (Fig. 9(b) red circle area).

This also explains why MSAR-BM3D has lower PSNR values. In addition, some of the textures in MSAR-BM3D results are over smoothed, such as the blue circle area in Fig. 9(b).

Similar phenomena can be observed on the TerraSAR-X images presented Fig. 10. Whereas Fig. 10(c) and (d) visually provide satisfying results and homogeneous residual noise images, both 2SPPB and MSAR-BM3D smoothed out some 


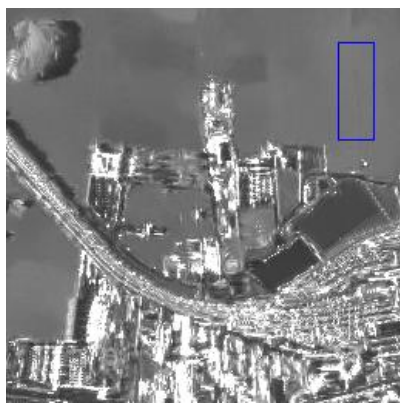

(a)

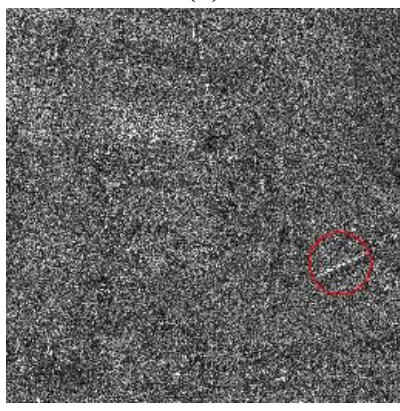

(e)

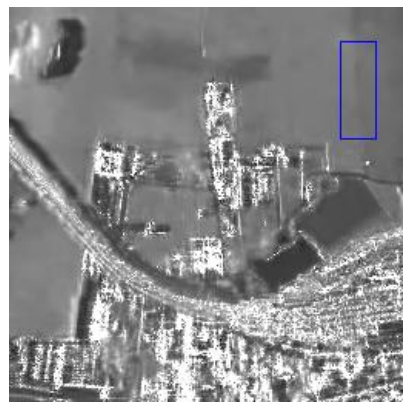

(b)

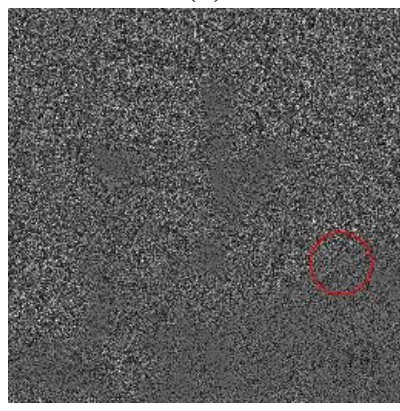

(f)

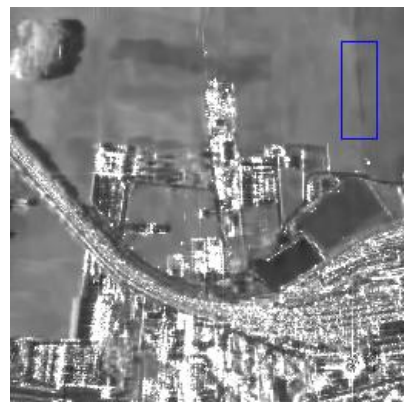

(c)

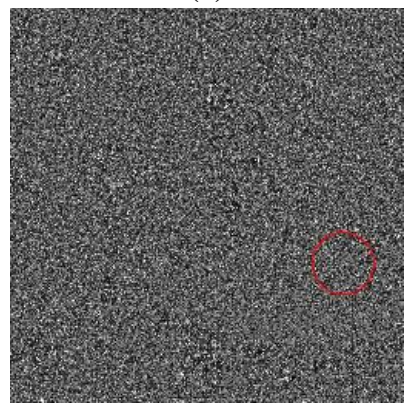

(g)

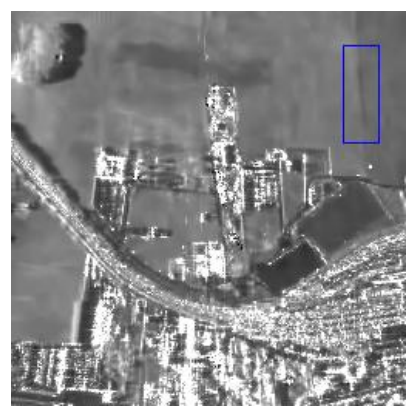

(d)

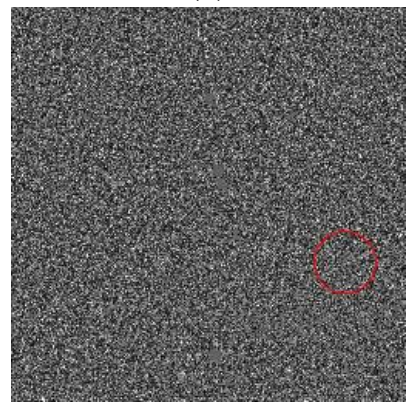

(h)

Fig. 10: Denoising results of TerraSAR-X images over Saint-Gervais (using Rulog). The original noisy image is available in Fig. 4(f). (a) 2SPPB, (b) MSAR-BM3D, (c) RABASAR-DAM, (d) RABASAR-DBWAM, (e-h) residual noise images (ratio of noisy image and denoised results). 26 TerraSAR-X images are used.

textures (Fig. 10 blue circle areas), or keep unchanged some noisy areas.

\section{CONCLUSION AND FUTURE WORK}

This paper has proposed a ratio-based multi-temporal denoising framework. During the restoration of each SAR image, it exploits the temporal information through a super-image. The use of different strategies to compute the super-images has been analyzed. RABASAR can provide better PSNR and MSSIM values when using a spatially denoised super-image. With the increase of the number of images in the time series, the differences of using different super-images decrease. When there are changes in the time series, using a binary weighted arithmetic mean can also provide good results. Based on the processing of time-series corrupted by simulated speckle noise, actual Sentinel-1 stacks and TerraSAR-X stacks, the qualitative and quantitative comparisons with UTA, NLTF, MSAR-BM3D and 2SPPB methods showed the potential of RABASAR to better preserve structures in multi-temporal SAR images while efficiently removing speckle. Besides, the super-image can be easily updated when a new data becomes available so as to process new images on-line.

Future work will be devoted to the updating framework, specially for the "re-computation" of the super-image and to the further processing of denoised time series.

\section{APPENDIX}

\section{DISTRIBUTION OF THE RATIO IMAGE}

In this appendix we present a simple way of deriving the distribution (pdf) followed by the ratio $\tau_{t}=\frac{v_{t}}{\hat{u}_{m}}$. The pdf of the ratio of 2 gamma-distributed random variables has been established in [43], and used in [44] for edge detection in SAR images. Following [45] [46], the Mellin framework allows a straightforward derivation.

We assume that the numerator $v_{t}$ follows a gamma distribution $\mathcal{G}\left(u_{t}, L\right)$, the denominator $\hat{u}_{m}$ follows a gamma distribution $\mathcal{G}\left(u_{m}, L_{m}\right)$, and that $v_{t}$ and $\hat{u}_{m}$ are independent. We will denote by $\hat{x}$ the Mellin convolution, and $\mathcal{H}(u)$ the homothetic pdf defined by $\mathcal{H}(u)(\nu)=\frac{1}{u} \delta\left(\frac{\nu}{u}-1\right)$. We have the following relationship between a gamma distributed pdf of mean 1 and a gamma pdf of mean $u_{t}$ [35]:

$$
\mathcal{G}\left(u_{t}, L\right)=\mathcal{H}\left(u_{t}\right) \hat{\times} \mathcal{G}(1, L)
$$

In the same way, we have the following relation for the pdf of $\hat{u}_{m}$ :

$$
\mathcal{G}\left(u_{m}, L_{m}\right)=\mathcal{H}\left(u_{m}\right) \hat{\times} \mathcal{G}\left(1, L_{m}\right)
$$

The variable $\hat{u}_{m}^{-1}$ follows an inverse gamma distribution $\mathcal{G I}$ given by:

$$
\begin{aligned}
& p_{\hat{u}_{m}^{-1}}\left(\hat{u}_{m}^{-1} \mid u_{m}, L_{m}\right)=\left[\mathcal{H}\left(u_{m}^{-1}\right) \hat{\times} \mathcal{G I}\left(1, L_{m}\right)\right]\left(\hat{u}_{m}^{-1}\right) \\
& \text { with } \mathcal{G I}(\mu, M)(\nu)=\frac{1}{\Gamma(M)} \frac{1}{M \mu}\left(\frac{M \mu}{\nu}\right)^{(M+1)} e^{-\frac{M \mu}{\nu}}
\end{aligned}
$$

Multiplying the variables $v_{t}$ and $\frac{1}{\hat{u}_{m}}$, we thus have the Mellin convolution of $\mathcal{H}\left(\frac{u_{t}}{u_{m}}\right), \mathcal{G}(1, L)$ and $\mathcal{G} \mathcal{I}\left(1, L_{m}\right)$ which leads 


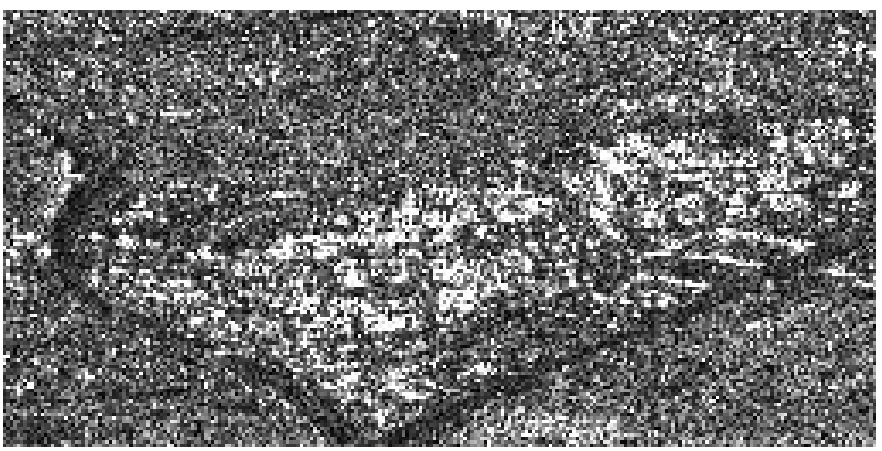

(a) Noisy image

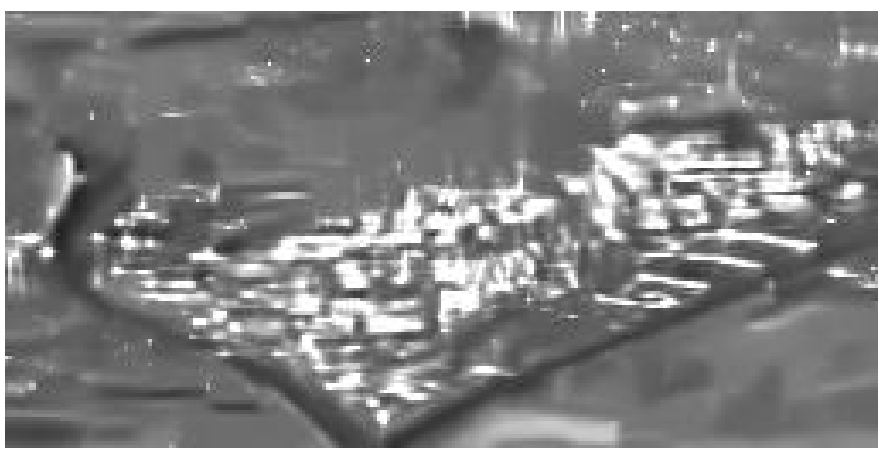

(b) MuLoG-BM3D

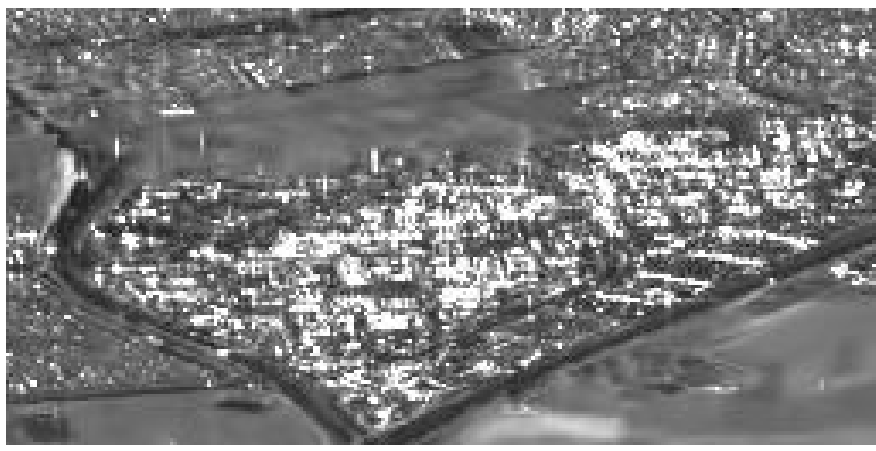

(c) RABASAR-DAM

Fig. 11: Noisy image (a) and denoising results (b-c) of MuLoG-BM3D and RABASAR-DAM. 64 resampled Sentinel-1 images are used to calculate the arithmetic mean image.

to a Fisher pdf $\mathcal{F}\left(\rho_{t}, L, L_{m}\right)$ [35] [46], noting $\rho_{t}=\frac{u_{t}}{u_{m}}$ :

$$
\begin{aligned}
p_{\tau_{t}}\left(\tau_{t} \mid \rho_{t}, L, L_{m}\right) & =\left[\mathcal{H}\left(\rho_{t}\right) \hat{\times} \mathcal{G}(1, L) \hat{\times} \mathcal{G} \mathcal{I}\left(1, L_{m}\right)\right]\left(\tau_{t}\right) \\
& =\left[\mathcal{H}\left(\rho_{t}\right) \hat{\times} \mathcal{F}\left(1, L, L_{m}\right)\right]\left(\tau_{t}\right) \\
& =\mathcal{F}\left(\rho_{t}, L, L_{m}\right)\left(\tau_{t}\right) \\
& =\frac{L}{L_{m}} \frac{1}{\rho_{t}} \frac{\Gamma\left(L+L_{m}\right)}{\Gamma(L) \Gamma\left(L_{m}\right)} \frac{\left(\frac{L}{L_{m}} \frac{\tau_{t}}{\rho_{t}}\right)^{L-1}}{\left(1+\frac{L}{L_{m}} \frac{\tau_{t}}{\rho_{t}}\right)^{\left(L+L_{m}\right)}}
\end{aligned}
$$

\section{ACKNOWLEDGMENT}

The authors would like to thank the CNES (French Space Agency, project DAJ/AR/IB-2016-10117102) and the China Scholarship Council for their fundings.

\section{REFERENCES}

[1] W. Zhao, C. Deledalle, L. Denis, H. Maître, J. Nicolas, and F. Tupin "RABASAR: A fast ratio based multi-temporal SAR despeckling," in IEEE International Geoscience and Remote Sensing Symposium, IGARSS 2018, 2018.

[2] F. Argenti, A. Lapini, T. Bianchi, and L. Alparone, "A tutorial on speckle reduction in Synthetic Aperture Radar images," IEEE Geoscience and remote sensing magazine, vol. 1, no. 3, pp. 6-35, 2013.

[3] R. Touzi, "A review of speckle filtering in the context of estimation theory," IEEE Transactions on Geoscience and Remote Sensing, vol. 40, no. 11, pp. 2392-2404, 2002.

[4] C. Deledalle, L. Denis, G. Poggi, F. Tupin, and L. Verdoliva, "Exploiting patch similarity for SAR image processing: the nonlocal paradigm," IEEE Signal Processing Magazine, vol. 31, no. 4, pp. 69-78, 2014.

[5] J. Lee, "Speckle analysis and smoothing of Synthetic Aperture Radar images," Computer graphics and image processing, vol. 17, no. 1, pp. 24-32, 1981

[6] _ _ "Refined filtering of image noise using local statistics," Computer graphics and image processing, vol. 15, no. 4, pp. 380-389, 1981.

[7] P. Meer, Park, R.H., and K. Cho, "Multiresolution adaptive image smoothing," CVGIP: Graphical Models and Image Processing, vol. 56, no. 2, pp. 140-148, 1994.

[8] G. Vasile, E. Trouvé, J.-S. Lee, and V. Buzuloiu, "Intensity-driven adaptive-neighborhood technique for polarimetric and interferometric SAR parameters estimation," IEEE Transactions on Geoscience and Remote Sensing, vol. 44, no. 6, pp. 1609-1621, 2006.

[9] C. Deledalle, L. Denis, and F. Tupin, "Iterative weighted maximum likelihood denoising with probabilistic patch-based weights," IEEE Transactions on Image Processing, vol. 18, no. 12, pp. 2661-2672, 2009.

[10] C. Deledalle, L. Denis, F. Tupin, Reigber, and M. A. and Jäger, "NLSAR: A unified nonlocal framework for resolution-preserving (Pol)(In) SAR denoising," IEEE Transactions on Geoscience and Remote Sensing, vol. 53, no. 4, pp. 2021-2038, 2015.

[11] S. Foucher, "SAR image filtering via learned dictionaries and sparse representations," in Geoscience and Remote Sensing Symposium, 2008. IGARSS 2008. IEEE International, vol. 1. IEEE, 2008, pp. I-229.

[12] S. Parrilli, M. Poderico, C. Angelino, and L. Verdoliva, "A nonloca SAR image denoising algorithm based on LLMMSE wavelet shrinkage," IEEE Transactions on Geoscience and Remote Sensing, vol. 50, no. 2, pp. 606-616, 2012.

[13] K. Zhang, W. Zuo, Y. Chen, D. Meng, and L. Zhang, "Beyond a gaussian denoiser: Residual learning of deep CNN for image denoising," IEEE Transactions on Image Processing, vol. 26, no. 7, pp. 3142-3155, 2017.

[14] G. Chierchia, D. Cozzolino, G. Poggi, and L. Verdoliva, "SAR image despeckling through convolutional neural networks," arXiv preprint arXiv:1704.00275, 2017.

[15] P. Wang, H. Zhang, and V. Patel, "SAR image despeckling using a convolutional neural network," IEEE Signal Processing Letters, vol. 24 no. 12 , pp. $1763-1767,2017$.

[16] A. Moreira, P. Prats-Iraola, M. Younis, G. Krieger, I. Hajnsek, and K. Papathanassiou, "A tutorial on synthetic aperture radar," IEEE Geoscience and Remote Sensing Magazine, vol. 1, no. 1, pp. 6-43, 2013.

[17] J. Lee, M. Grunes, and S. Mango, "Speckle reduction in multipolarization, multifrequency SAR imagery," IEEE Transactions on Geoscience and Remote Sensing, vol. 29, no. 4, pp. 535-544, 1991.

[18] S. Quegan, T. Le Toan, J. Yu, F. Ribbes, and N. Floury, "Multitemporal ERS SAR analysis applied to forest mapping," IEEE Transactions on Geoscience and Remote Sensing, vol. 38, no. 2, pp. 741-753, 2000.

[19] S. Quegan and J. Yu, "Filtering of multichannel SAR images," IEEE Transactions on Geoscience and Remote Sensing, vol. 39, no. 11, pp. 2373-2379, 2001.

[20] D. Coltuc, E. Trouvé, F. Bujor, N. Classeau, and J. Rudant, "Time-space filtering of multitemporal SAR images," In Geoscience and Remote Sensing Symposium, Proceedings. IGARSS 2000., vol. 7, pp. 2909-2911, 2000.

[21] G. Chierchia, M. El Gheche, G. Scarpa, and L. Verdoliva, "Multitemporal SAR image despeckling based on block-matching and collaborative filtering," IEEE Transactions on Geoscience and Remote Sensing, 2017.

[22] T. Lê, A. Atto, E. Trouvé, and J. Nicolas, "Adaptive multitemporal SAR image filtering based on the change detection matrix," IEEE Geoscience and Remote Sensing Letters, vol. 11, no. 10, pp. 1826-1830, 2014.

[23] T. Lê, A. Atto, E. Trouvé, A. Solikhin, and V. Pinel, "Change detection matrix for multitemporal filtering and change analysis of SAR and PolSAR image time series," ISPRS Journal of Photogrammetry and Remote Sensing, vol. 107, pp. 64-76, 2015. 
[24] X. Su, C. Deledalle, F. Tupin, and H. Sun, "Two-step multitemporal nonlocal means for Synthetic Aperture Radar images," IEEE Transactions on Geoscience and Remote Sensing, vol. 52, no. 10, pp. 6181-6196, 2014.

[25] M. Ciuc, P. Bolon, E. Trouvé, V. Buzuloiu, and J. Rudant, "Adaptiveneighborhood speckle removal in multitemporal Synthetic Aperture Radar images," Applied Optics, vol. 40, no. 32, pp. 5954-5966, 2001.

[26] S. Lobry, L. Denis, and F. Tupin, "Multitemporal SAR image decomposition into strong scatterers, background, and speckle," IEEE Journal of Selected Topics in Applied Earth Observations and Remote Sensing, vol. 9, no. 8, pp. 3419-3429, 2016.

[27] J. Nicolas, E. Trouve, R. Fallourd, F. Vernier, F. Tupin, O. Harant, M. Gay, and L. Moreau, "A first comparison of Cosmo-Skymed and TerraSAR-X data over Chamonix Mont-Blanc test-site," In Geoscience and Remote Sensing Symposium (IGARSS), pp. 5586-5589, Jul. 2012.

[28] C. Deledalle, L. Denis, S. Tabti, and F. Tupin, "MuLoG, or how to apply Gaussian denoisers to multi-channel SAR speckle reduction?" IEEE Transactions on Image Processing, vol. 26, no. 9, pp. 4389-4403, 2017.

[29] X. Su, C. Deledalle, F. Tupin, and H. Sun, "NORCAMA: Change analysis in SAR time series by likelihood ratio change matrix clustering," ISPRS Journal of Photogrammetry and Remote Sensing, vol. 101, pp. 247-261, 2015.

[30] G. Quin, B. Pinel-Puyssegur, J. Nicolas, and P. Loreaux, "MIMOSA An automatic change detection method for SAR time series," IEEE Transactions on Geoscience and Remote Sensing, vol. 52, no. 9, pp. 5349-5363, 2014

[31] J. Goodman, Speckle phenomena in optics: theory and applications. Roberts and Company Publishers, 2007.

[32] C. Oliver and S. Quegan, Understanding Synthetic Aperture Radar images. SciTech Publishing, 2004.

[33] C. Deledalle, L. Denis, and F. Tupin, "How to compare noisy patches? patch similarity beyond gaussian noise," International journal of computer vision, vol. 99, no. 1, pp. 86-102, 2012.

[34] C. Tison, J. Nicolas, F. Tupin, and H. Maître, "A new statistical model for markovian classification of urban areas in high-resolution SAR images," IEEE Transactions on Geoscience and Remote Sensing, vol. 42, no. 10, pp. 2046-2057, 2004.

[35] J. Nicolas, "Introduction to second kind statistics: Application of logmoments and log-cumulants to the analysis of radar image distributions," Traitement du Signal (translated by S. Anfinsen), vol. 19, no. 3, pp. 139167, 2002.

[36] J. M. Bioucas-Dias and M. A. Figueiredo, "Multiplicative noise removal using variable splitting and constrained optimization," IEEE Transactions on Image Processing, vol. 19, no. 7, pp. 1720-1730, 2010.

[37] R. Fisher, "On a distribution yielding the error functions of several well known statistics," in Proceedings of the International Congress of Mathematics, 1924.

[38] S. H. Chan, X. Wang, and O. A. Elgendy, "Plug-and-play ADMM for image restoration: Fixed point convergence and applications," IEEE Transactions on Computational Imaging, 2016.

[39] K. Dabov, A. Foi, V. Katkovnik, and K. Egiazarian, "Image denoising by sparse 3-D transform-domain collaborative filtering," IEEE Transactions on image processing, vol. 16, no. 8, pp. 2080-2095, 2007.

[40] R. Abergel, L. Denis, S. Ladjal, and F. Tupin, "Sidelobes suppression and strong targets extraction in single look complex SAR images with several applications," IEEE Journal of Selected Topics in Applied Earth Observations and Remote Sensing, vol. 11, no. 3, pp. 759-776, 2018.

[41] Z. Wang, A. Bovik, H. Sheikh, and E. Simoncelli, "Image quality assessment: from error visibility to structural similarity," IEEE Transactions on Image Processing, vol. 13, no. 4, pp. 600-612, 2004.

[42] A. Buades, B. Coll, and J. Morel, "A non-local algorithm for image denoising," in Computer Vision and Pattern Recognition, 2005. CVPR 2005. IEEE Computer Society Conference on, vol. 2. IEEE, 2005, pp. 60-65.

[43] H. Cramer, "Mathematical methods of statistics," in Princeton University Press, 1946.

[44] R. Touzi, A. Lopes, and P. Bousquet, "A statistical and geometrical edge detector for SAR images," IEEE Transactions on Geoscience and Remote Sensing, vol. 26, no. 6, pp. 764 - 773, Nov. 1988.

[45] B. Epstein, "Some applications of the Mellin transform in statistics," Annals of Mathematical Statistics, no. 19, pp. 370-379, 1948.

[46] J. M. Nicolas, "A Fisher-MAP filter for SAR image processing," in IGARSS 2003. 2003 IEEE International Geoscience and Remote Sensing Symposium. Proceedings (IEEE Cat. No.03CH37477), vol. 3, July 2003, pp. 1996-1998. 